\title{
The Imprint of Vortical Structures on the Pressure Field at the Edge of a Turbulent High-Speed Jet
}

\author{
Andres Adam* and Dimitri Papamoschou ${ }^{\dagger}$ \\ University of California, Irvine, Irvine, CA, 92697, USA \\ Christophe Bogey \\ Univ Lyon, École Centrale de Lyon, INSA Lyon, Université Claude Bernard Lyon I, CNRS, F-69134, Ecully, \\ France
}

We investigate the distributions of length and velocity scales in a Mach 0.9 isothermal round jet for the purpose of developing a linear surface-based model for the noise source that can be informed by the time-averaged flow field. Large eddy simulation of the flow enables the computation of two-point space-time correlations throughout the jet and its near acoustic field. The resulting time, length, and convective-velocity scales are examined on the surface of peak Reynolds stress (SPS), representing the location of the most energetic eddies, and on a "radiator surface" at the boundary between the rotational and irrotational fields. The radiator surface is defined such that the convective velocity distribution on it matches that on the surface of peak Reynolds stress. The location of the radiator surface, and distributions of flow scales on it, are critical elements of the noise source model and are examined in detail. The nature of the space-time correlations, and resulting scales, differ significantly for axial velocity fluctuations and pressure fluctuations. Velocity-based correlations appear to capture localized turbulent events, while pressure-based correlations appear dominated by the interaction of large eddies with the surrounding potential flow. Consequently the axial and radial distributions of the corresponding length scales exhibit different trends. Correlation scales on the radiator surface are larger than on the SPS thus indicating that fine-scale vortical motions do not make a significant imprint on the radiator surface. Scales associated with the Reynolds-averaged flow field, relevant to low-cost modeling, are emulated from LES data and compared to the LES-based scales. Simple relationships are established that may aid the development of rapid predictive models.

$\begin{array}{lll}C & = & \text { proportionality factor } \\ D_{j} & = & \text { Nozzle exit diameter } \\ g & = & \text { principal component of the Reynolds stress } \\ k & = & \text { turbulence kinetic energy } \\ L & = & \text { fixed-frame length scale } \\ \widehat{L} & = & \text { moving-frame length scale } \\ M_{j} & = & \text { jet exit Mach number } \\ p & = & \text { pressure } \\ R & = & \text { space-time correlation }\end{array}$

\footnotetext{
*PhD Candidate, Department of Mechanical and Aerospace Engineering, aadamalb@uci.edu, AIAA Student Member.

${ }^{\dagger}$ Professor, Department of Mechanical and Aerospace Engineering, dpapamos@uci.edu, AIAA Fellow.

¥CNRS Research Scientist, Laboratoire de Mécanique des Fluides et d’Acoustique, UMR CNRS 5509, christophe.bogey@eclyon.fr, AIAA Associate Fellow.
} 


$\begin{array}{lll}r_{i} & = & \text { radial location of inner edge of mixing layer } \\ S t_{D} & = & \text { Strouhal number based on nozzle diameter } \\ T & = & \text { temperature } \\ t & = & \text { time } \\ U_{j} & = & \text { jet exit velocity } \\ U_{c} & = & \text { convective velocity } \\ \bar{u}_{\mathrm{CL}} & = & \text { centerline time-averaged velocity } \\ u, v, w & = & \text { velocity components in Cartesian coordinate system } \\ x, r, \phi & = & \text { cylindrical coordinates, with } x \text { on jet axis } \\ x, y, z & =\text { Cartesian coordinates, with } x \text { on jet axis } \\ \delta_{\theta} & =\text { momentum thickness of the mixing layer } \\ \xi, \eta, \alpha & =\text { displacements in cylindrical coordinates } \\ \epsilon & = & \text { turbulence dissipation } \\ \Phi & = & \text { azimuthal scale } \\ \omega & = & \text { vorticity vector }\end{array}$

Superscripts

, $=$ temporal fluctuation

Acronyms

$\begin{array}{lll}\text { LES } & =\text { Large Eddy Simulation } \\ \text { SPS } & =\text { Surface of Peak Stress } \\ \text { RANS } & =\text { Reynolds-Averaged Navier Stokes }\end{array}$

\section{Introduction}

The research effort presented here is motivated by the need of the aerospace community for low-order predictive models of the jet noise emission. For these models to have satisfactory turnaround times and become useful design tools, they need to rely on low-cost solutions of the flow field such as Reynolds-Averaged Navier Stokes (RANS) simulations. The focus is on surface-based models which constitute an alternative to the acoustic analogy models started with Lighthill [1]. In surface-based models, the noise source is prescribed on a surface surrounding the jet flow, commonly located in the linear pressure field [2, 3]. This approach has the advantage of allowing predictions of not only propagation but also scattering from nearby objects (i.e. airframe surfaces) using well known linear propagation techniques such as the boundary element method [4-6]. In addition, surface-based models could simplify the treatment of complex multi-stream asymmetric jets that have shown promising noise reductions [7, 8]. To be able to inform the surface-based noise source model from the RANS flow field one needs to establish physical connections between the dominant turbulent events in the vortical region of the jet and the linear pressure statistics near the edge of the jet where the surface-based model is defined. The basic elements of this connection, as proposed by Ref. [9], are depicted in Fig. 1. It is envisioned that the action of the turbulent eddies that dominate sound production is represented by the flow statistics on the surface of peak Reynolds stress, abbreviated here as SPS. It is on this surface where the information on the vortical field would be extracted from the RANS solution. The noise source is prescribed as a distribution of random partial fields on a "radiator surface" at the edge of the jet, defined as the boundary between the rotational flow field and the outer linear pressure field. Each partial field is constituted by an amplitude modulated traveling wave with finite axial and azimuthal length scales. Critical elements of the partial fields include their location, convective velocity $U_{c}$, and correlation scales in the axial 


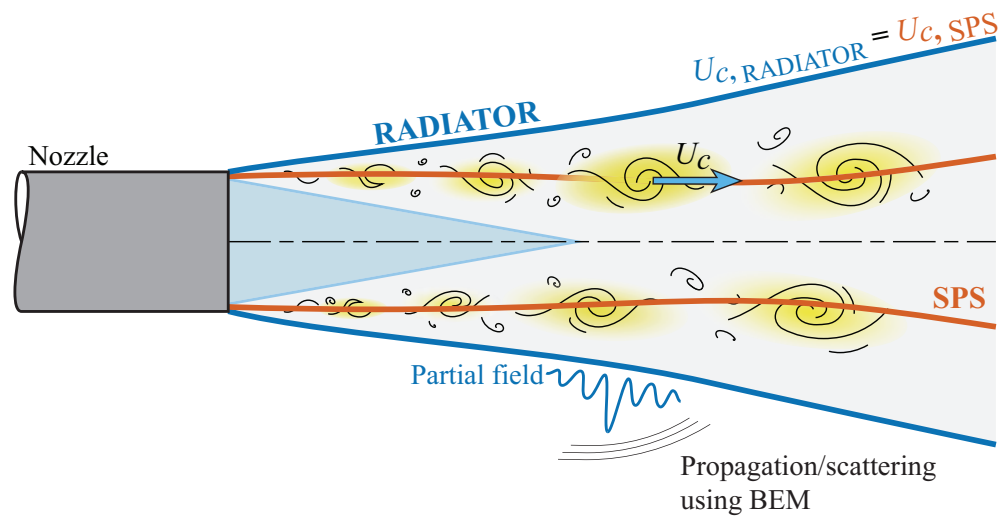

Fig. 1 Basic elements of surface-based modeling.

and azimuthal directions. Previous works [10,11] have studied the distribution of convective velocity $U_{c}$, as calculated from space-time correlations, and have found two regions: an inner region, encompassing the jet flow, where $U_{c}$ generally follows the trend of mean axial velocity and thus decays radially; and an outer region, near the edge of the jet and beyond, where $U_{c}$ rapidly increases to supersonic values indicative of the flow field. The same works proposed that a suitable placement of the radiator surface is at the region where $U_{c}$, based on pressure fluctuations, matches the convective velocity of the turbulence structures on the SPS based on velocity fluctuations. It was also found that $U_{c}$ on the SPS can be accurately modeled as the mean axial velocity on the SPS, therefore allowing its determination from the RANS flow field.

Turbulence scales in jet shear layers have received considerable attention in the literature. Among others, one can outline the measurements of Harper-Bourne [12] and Morris and Zaman [13], which are frequently used to inform jet noise source models. More recently, Proença et al. [14] performed a comprehensive hot-wire investigation in the shear layer of various unheated single-stream jets. However, past works have focused primarily on the jet lip line and centerline and provide little information on turbulent scales near the edge of the jet. This motivates the present work, where we seek physical connections between flow scales in the vortical field and at the edge of the jet.

In this paper, we analyze a high-definition large eddy simulation (LES) of a single-stream jet at Mach 0.9. Using two-point space-time correlations we calculate characteristic length and velocity scales throughout the jet flow and in the near acoustic field. The distribution of these scales on the SPS and on the radiator surface are of particular interest. We thus aim to investigate physical connections between the core of the vortical field and the resulting pressure signature at the edge of the jet where a linear source model would be prescribed. In addition we use the LES results to emulate the RANS flow field and evaluate the effectiveness of using RANS to model the scales relevant to noise generation.

\section{Numerical Flow Field}

\section{A. Jet Flow}

We consider an isothermal, single-stream round jet at velocity $U_{j}=309 \mathrm{~m} / \mathrm{s}$, Mach number $M_{j}=0.9$ and diameter-based based Reynolds number $\operatorname{Re}_{D}=10^{5}$. The jet originates from a pipe of constant diameter $D_{j}$ and discharges into air with temperature $T_{a}=293 \mathrm{~K}$ and pressure $p_{a}=10^{5} \mathrm{~Pa}$. The pipe exit is at $x=0$, and the pipe wall thickness is $0.053 D_{j}$. This jet has been studied in previous works [15-18].

The pipe flow originates at $x=-D_{j}$, where a Blasius laminar boundary-layer profile of thickness 
$\delta_{B L}=0.075 D_{j}$ is imposed for the axial velocity. In addition, radial and azimuthal velocities are set to zero, pressure is equal to its ambient value, and the temperature is resolved by a Crocco-Busemann relation. The jet is initially disturbed by adding random low-level vortical structures uncorrelated in the azimuthal direction at $x=-0.475 D_{j}$ inside the pipe [15]. The forcing strength is empirically set to match the nozzle-exit conditions measured in a tripped jet by Zaman [19]. The resulting mean axial velocity profile is very similar to the laminar profile imposed at the nozzle inlet, while the rms velocity profile shows peak axial turbulence intensity $u_{e}^{\prime} / U_{j}=0.0914$.

\section{B. Numerical Methods}

The numerical framework for the LES used in this work is identical to that used in previous jet simulations [15-18, 20,-23]. The computational code solves the three-dimensional Navier-Stokes equations in cylindrical coordinates $(x, r, \phi)$ using low-dissipation and low-dispersion explicit schemes. The singularity at the axis is solved by the method of Mohseni and Colonius [24]. The region near the axis also presents a restriction in time-step that is alleviated by calculating the azimuthal derivatives at coarser resolutions than those permitted by the grid [25], yielding an azimuthal resolution of $2 \pi / 16$. Spatial discretization is performed by fourth-order eleven-point centered finite differences, and time integration is carried out using a second-order six-stage Runge-Kutta algorithm [26]. The flow variables are filtered every time step by a sixth-order eleven-point centered explicit filter [27].

The explicit filtering used in the present simulation has the dual purpose of removing grid-to-grid oscillations and of performing as a subgrid-scale high-order dissipation model for the regions where the mesh grid is not fine enough to capture the smallest scales of turbulence.

Filters and non-centered finite differences also take place near the pipe walls and grid boundaries. At the boundaries, a sponge zone combining grid stretching and Laplacian filtering [28] is used to avoid acoustic reflections, in addition to the radiation conditions of Tam and Dong [29].

The effectiveness of this numerical framework has been assessed in previous studies in diverse cases such as subsonic jets, Taylor-Green vortices and turbulent channel flows [15, 30,-33], where it has been compared against direct numerical simulations and the properties of the filtering dissipation have been examined.

\section{Simulation Parameters}

The simulation presented is a highly-resolved LES. Its characteristics are reported in detail in a recent paper by Bogey [17], where a grid-sensitivity study of the jet flow and acoustic fields is carried out. The mesh grid contains $n_{x} \times n_{\phi} \times n_{r}=2085 \times 1024 \times 512$ points, and its physical extents are $20 D_{j}$ and $7.5 D_{j}$ in the axial and radial dimensions respectively. The minimum mesh spacings are equal to $\Delta r=0.0018 D_{j}$ at $r=D_{j} / 2$ and $\Delta x=0.0036 D_{j}$ at $x=0$. The maximal mesh spacing in the jet near field is equal to $\Delta r=0.0375$, leading to a Strouhal number of $S t_{D}=f D_{j} / U_{j}=5.9$ for an acoustic wave discretized by five points per wavelength, where $f$ is the frequency.

The simulations have been performed with an OpenMP-based in-house solver, using a time step $\Delta t=0.7 \times \Delta r_{\text {min }} / c_{0}$ to ensure numerical stability. The simulation time after the transient period is equal to $1250 D_{j} / U_{j}$. During the stationary time, density, velocity components and pressure are recorded at a sampling frequency of $S t_{D}=6.4$ in eight equidistant azimuthal angles. Their Fourier coefficients in the azimuthal directions, estimated over the full section $(x, r)$, are also saved for the first nine azimuthal modes. The statistics are averaged in the azimuthal direction, when possible. The size of the database amounts to approximately $6.5 \mathrm{~TB}$. 


\section{Definition of Flow Scales}

\section{A. LES-based scales}

\section{Space-time correlations}

In the present work, the measures of length scales are surveyed using two-point space-time correlations given a reference position in the domain. The normalized space-time correlation of variable $f$ in the meridian section $(x, r)$ is defined as

$$
R_{f f}(x, r ; \xi, \eta, \tau)=\frac{\overline{f(x, r, t) f(x+\xi, r+\eta, t+\tau)}}{\overline{f(x, r)}^{1 / 2} \overline{f(x+\xi, r+\eta)}^{1 / 2}}
$$

where $\xi$ is the displacement in the axial direction, $\eta$ is the displacement in the radial direction, $\tau$ is the time shift, and overline denotes time averaging. The variable $f$ can correspond to the fluctuating axial velocity or pressure, yielding $R_{u u}$ or $R_{p p}$ respectively.

The values of variables $\xi$ and $\eta$ of Eq. 1 are linked so that the displacement angle $\theta=\arctan (\eta / \xi)$ is consistent with the flow spreading, shown schematically in Fig. 2. At points below the lip line $r=0.5 D_{j}$, the displacement is axial. Above the lip line, the displacement aligns with the reference location $(x, r)$ and the nozzle lip, up to a maximum of 9 degrees. The choice of limiting the displacement angle to 9 degrees comes after analyzing the geometry of the radiator surface explained in section IV, which expands almost uniformly at that value.

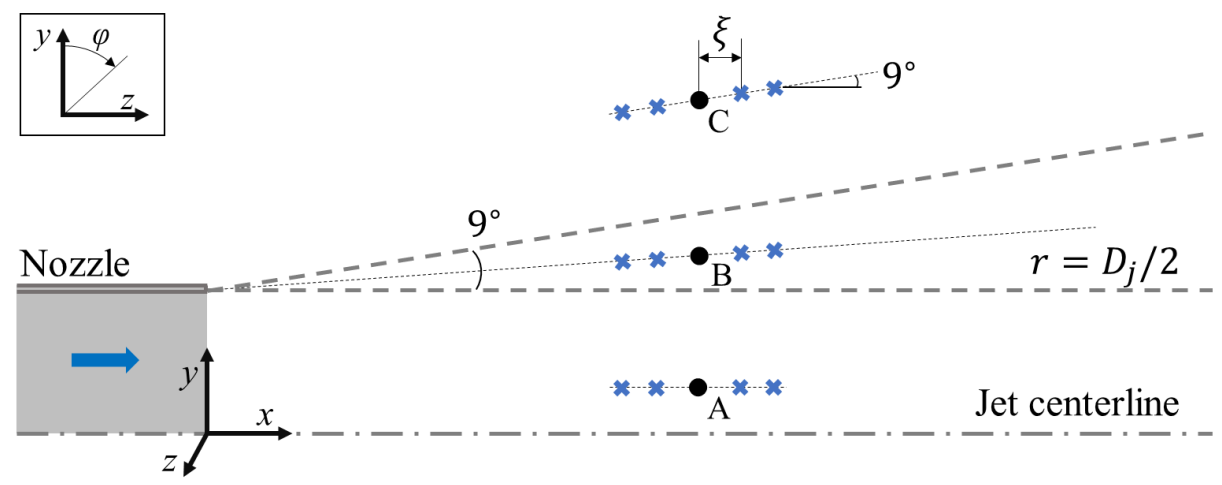

Fig. 2 Schematic drawing of displacement angle on two-point correlations. Black circles: examples of reference locations $(x, r)$. Blue crosses: examples of displacements $(x+\xi, r+\eta)$. Label A: point below lip line. Label B: point above lip line, below $9^{\circ}$ to lip edge. Label C: point above $9^{\circ}$ to lip edge.

\section{Fixed-frame length scales}

The fixed-frame length scale is the axial distance $L$ over which events remain correlated for a fixed time frame. Following Harper-Bourne [12], Morris and Zaman [13], and Dahl [34], we define the length scale as the displacement $\xi$ at which the correlation $R_{f f}$ decays to $1 / \mathrm{e}$. We also note that $R_{f f}$ is not an even function with $\xi$ [14], so displacements $\xi$ are taken in the negative and positive directions relative to the reference location. This method yields two displacements $L_{f_{1}}<0$ and $L_{f_{2}}>0$ that satisfy

$$
R_{f f}\left(\xi=L_{f_{i}}, \tau=0\right)=1 / \mathrm{e}, \quad(i=1,2)
$$




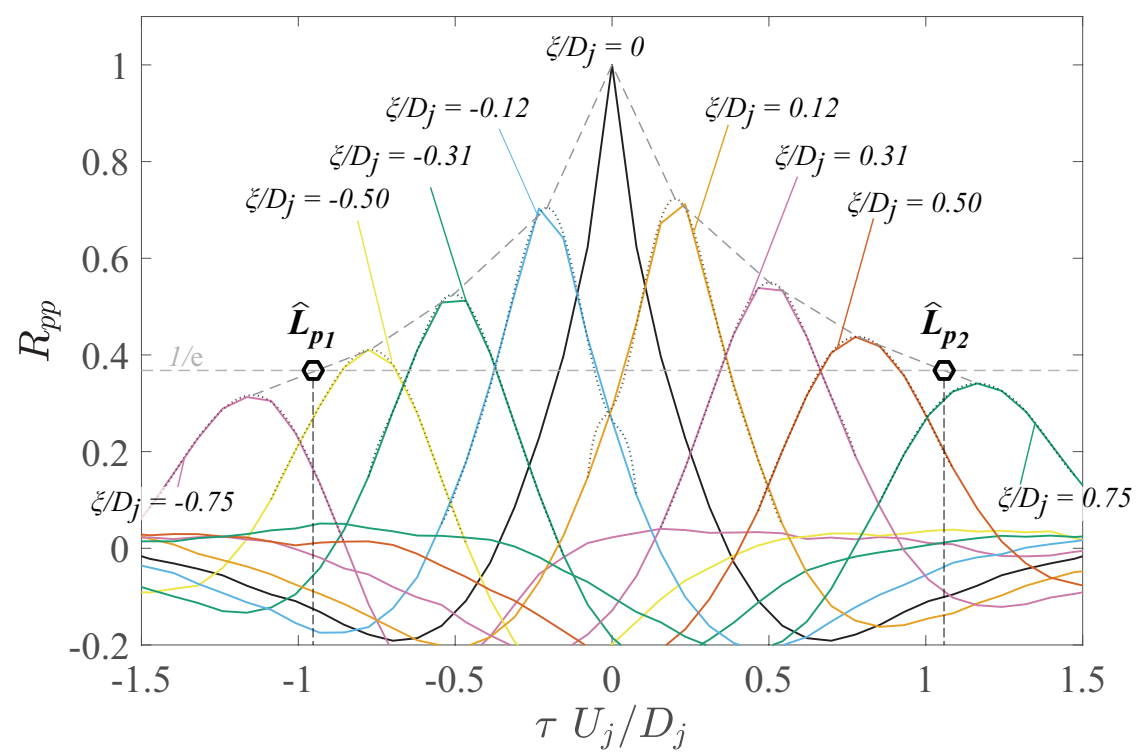

Fig. 3 Space-time correlation $R_{p p}$ at $x / D_{j}=2.5$ and $r / D_{j}=0.5$. Dotted lines: fits by seventh-order polynomials. Dashed lines: relevant marks for the calculation of $\widehat{L}_{p}$.

where for brevity we omit the coordinates of the reference location from the argument of $R_{f} f$. We define the fixed-frame length scale as the average of the magnitude of the two displacements:

$$
L_{f}=\frac{1}{2}\left(\left|L_{f_{1}}\right|+L_{f_{2}}\right)
$$

In practice the application of Eq. 2 requires interpolation between the discrete data of the simulation. The definition of length scale used here differs from the classical method of calculating the length scale as the integral of the correlation curve [35]. The two approaches yield similar results as the integral approach often terminates at the first zero crossing. We carried out comparisons of the two approaches and found that, while the quantitative values differ slightly, the qualitative trends are consistent.

\section{Moving-Frame Length Scales}

We consider an additional axial length scale, the moving-frame length scale $\widehat{L}$. This scale is a measure of the axial distance over which turbulence remains correlated as it convects downstream. It can also be interpreted as the "length of life" of a turbulent eddy, the distance traveled until its characteristics are lost through pairing or other mechanisms. Kerhervé et al. [35] note that this is not a "real" length scale, but rather a product of the convective velocity and a time scale. Nevertheless, it is an intrinsic characteristic of the stochastic events of the jet and can be useful for source modeling. Similarly to the fixed-frame length scales, we compute two values $\widehat{L}_{f_{1}}<0$ and $\widehat{L}_{f_{2}}>0$ for negative and positive displacements, respectively, around a reference location that satisfy

$$
R_{f f}\left(\xi=\widehat{L}_{f_{i}}, \tau=\tau_{R_{\max }}\right)=1 / \mathrm{e}, \quad(i=1,2)
$$

where $\tau_{R_{\max }}$ is the time delay that maximizes the correlation $R_{f f}$ for a reference location and displacement $\xi$. The moving-frame length scale is then defined as

$$
\widehat{L}_{f}=\frac{1}{2}\left(\left|\widehat{L}_{f_{1}}\right|+\widehat{L}_{f_{2}}\right)
$$


In practice $\widehat{L}_{f}$ is calculated by considering the envelope of space-time correlations at various axial separations $\xi_{i}$. Figure 3 shows an example of space-time correlations based on pressure fluctuation $p^{\prime}$ for a reference location in the vortical field. The values of $\xi$ corresponding to $\widehat{L}_{f_{1}}$ and $\widehat{L}_{f_{2}}$ are linearly interpolated from the correlation maxima. In order to improve the precision of the method, the exact locations of the those maxima are calculated by fitting seventh-order polynomials to the correlation curves.

\section{Convective Velocity}

The space-time correlations are also used to calculate the convective velocity of fluctuations everywhere in the domain. The convective velocity for a given axial separation $\xi_{i}$, is $U_{c_{i}}=\xi_{i} / \tau_{R_{\max , i}}$. The final convective velocity at a reference location is calculated as the average of all $U_{c_{i}}$ extracted from correlations whose peak correlation exceeds 0.4 . For the calculation of convective velocity, the radial displacement is $\eta=0$. Therefore, the convective velocity presented here relates to the axial motion of events. Similarly to the flow scales, the convective velocity can be based on velocity or pressure fluctuations, yielding $U_{c_{u}}$ and $U_{c_{p}}$, respectively.

\section{Azimuthal Scales}

The azimuthal scale is angular and is defined at fixed axial, radial, and time separations. The related two-point correlation is

$$
R_{f f}(x, r, \phi ; \alpha)=\frac{\overline{f(x, r, \phi, t) f(x, r, \phi+\alpha, t)}}{\overline{f(x, r, \phi)}^{1 / 2} \overline{f(x, r, \phi+\alpha)}^{1 / 2}}
$$

where $\alpha$ is the the azimuthal separation. For a given reference point $(x, r, \phi)$, the azimuthal scale is defined by

$$
R_{f f}\left(\alpha=\Phi_{f}\right)=1 / \mathrm{e}
$$

The range of the azimuthal scale is $\Phi_{f} \in[0, \pi]$, where $\Phi_{f}=\pi$ indicates that $R_{f f}$ is above the threshold of $1 / \mathrm{e}$ for the entire azimuthal range. In this work, we reconstructed the azimuthal variation of the flow field from the first nine Fourier modes. This reconstruction captures most of the relevant flow features, but may fail to capture abrupt azimuthal variations that require a wider range of azimuthal modes. Future efforts will consider using the complete flow field.

\section{B. RANS-Based Scales}

RANS-based scales are relevant to low-cost modeling of the noise source. Here we do not use results directly derived from a RANS simulation of the same jet, but instead we use the statistics of the LES to "emulate" the results that a RANS simulation would yield. While we took this approach because of a lack of a readily available, equivalent RANS simulation, we can recognize an advantage of this "emulation" method: simulations by RANS and LES will show disparities in the flow field due to their difference in accuracy. Here, we can directly test the relation between turbulent structures and their impact on the time-averaged flow, without differences in the predicted flow fields. RANS-based flow scales rely on dimensional analysis of the time-averaged variables related to turbulence production and dissipation. Using temporarily the index notation $\left(x_{1}, x_{2}, x_{3}\right)=(x, y, z)$ and $\left(u_{1}, u_{2}, u_{3}\right)=(u, v, w)$, the turbulent kinetic energy is

$$
k=\frac{1}{2}\left(\overline{u_{1}^{\prime 2}}+\overline{u_{2}^{\prime 2}}+\overline{u_{3}^{\prime 2}}\right)
$$

and the dissipation is

$$
\epsilon=2 \bar{v} \overline{S_{i j}^{\prime} S_{i j}^{\prime}}
$$


where $v$ is the kinematic viscosity and

$$
S_{i j}^{\prime}=\frac{1}{2}\left(\frac{\partial u_{i}^{\prime}}{\partial x_{j}}+\frac{\partial u_{j}^{\prime}}{\partial x_{i}}\right)
$$

is the fluctuating rate of strain. A dimensional construct of $k$ and $\epsilon$ yields the RANS-based length scale

$$
L=C_{L} \frac{k^{3 / 2}}{\epsilon}
$$

where $C_{L}$ is an empirical coefficient. A RANS-based azimuthal scale can be defined similarly by dividing by the radial coordinate $r$,

$$
\Phi=C_{\Phi} \frac{k^{3 / 2}}{\epsilon r}
$$

LES allows for the direct computation of the turbulent kinetic energy. On the other hand, it does not allow for the direct evaluation of the dissipation because of the existence of the dissipation of the subgrid scale model, namely the filtering relaxation term in the present LES [31]. The "real" dissipation $\epsilon$ experienced by the flow, which would be the value given by a RANS simulation, must be computed through the budget of turbulence kinetic energy. Following Panchapakesan and Lumley [36], the dissipation on a turbulent axisymmetric jet is computed as

$$
\epsilon=-\left[\bar{u} \frac{\partial k}{\partial x}+\bar{v} \frac{\partial k}{\partial r}\right]-\left[\overline{v^{\prime 2}} \frac{\partial \bar{v}}{\partial r}+\overline{w^{\prime 2}} \frac{\bar{v}}{r}+\overline{u^{\prime 2}} \frac{\partial \bar{u}}{\partial x}+\overline{u^{\prime} v^{\prime}}\left(\frac{\partial \bar{v}}{\partial x}+\frac{\partial \bar{u}}{\partial r}\right)\right]-\frac{1}{2}\left[\frac{\partial \overline{\kappa v^{\prime}}}{\partial r}+\frac{\overline{\kappa v^{\prime}}}{r}+\frac{\partial \overline{\kappa u^{\prime}}}{\partial x}\right]
$$

where $\kappa=u^{\prime 2}+v^{\prime 2}+w^{\prime 2}$. We can identify the first term (in brackets) as advection, the second as production, and the third as turbulent diffusion of the turbulence kinetic energy.

\section{Relevant Surfaces}

\section{A. Surface of Peak Reynolds Stress}

In the acoustic analogy model of Ref. [37] it was surmised that the most representative region of the effects of noise-generating turbulence eddies is the outer-most peak of the magnitude of the principal component of the Reynolds stress

$$
g=\left|<u^{\prime} q^{\prime}>\right|
$$

where $u^{\prime}$ is the axial velocity fluctuation and $q^{\prime}$ is the traverse velocity fluctuation in the direction of the mean velocity gradient. Of course, for the case of a single-stream round jet there is only one peak of Reynolds stress $g$ and the mean velocity gradient is in the radial direction. The surface is therefore named SPS (Surface of Peak Stress). This surface is the region where the stochastic, highly energetic turbulence structures are expected to have a strong effect on the mean statistics on the flow. For this reason, the SPS is an important region for noise source modeling from low-cost simulations such as RANS [11].

\section{B. Radiator Surface}

The radiator surface is defined as a surface close to the jet axis on and outside of which the propagation of pressure perturbation is governed by the homogeneous linear wave equation. It is on this surface that the noise sources could be modeled in the form of linear partial fields [6]. This model would be informed by turbulence statistics of the vortical field computed by RANS. It is the purpose of this work to further understand the connection between the vortical field and radiator surface by studying their relation in turbulence scales. The specific definition used in the present work is the surface near the edge of the jet where the $p^{\prime}$-based convective velocity matches the $u^{\prime}$-based convective velocity on the SPS at the same axial location. This definition has been used by previous works on multi-stream jets [10, 11]. 


\section{Overview of the Flow Field}

In the following we present contour plots that highlight the flow characteristics and positions of the relevant surfaces. Figure 4 plots the normalized mean axial velocity, $\bar{u} / U_{j}$, averaged in the azimuthal direction. The SPS is plotted as the red line, located within the shear layer, near the peak of the radial gradient of mean velocity. The radiator surface is positioned at the edge of the jet, in a region of very low velocity, and grows in an approximately linear fashion.

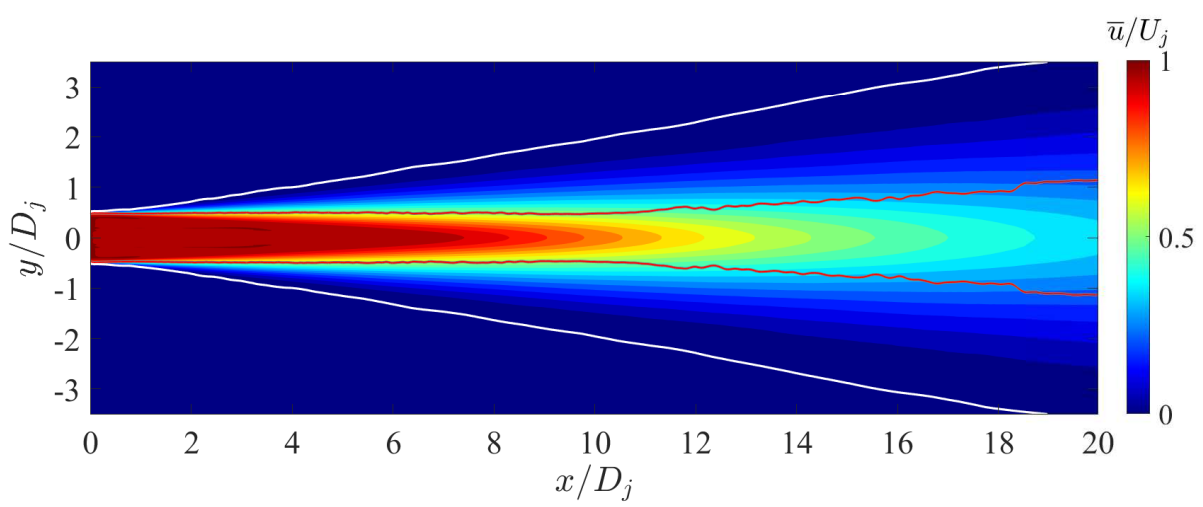

Fig. 4 Isocontours of normalized mean axial velocity $\bar{u} / U_{j}$. Red line: SPS. White line: radiator surface.

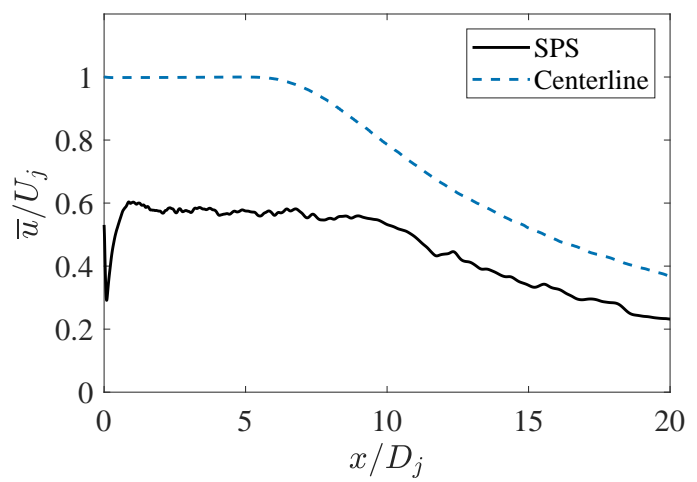

(a)

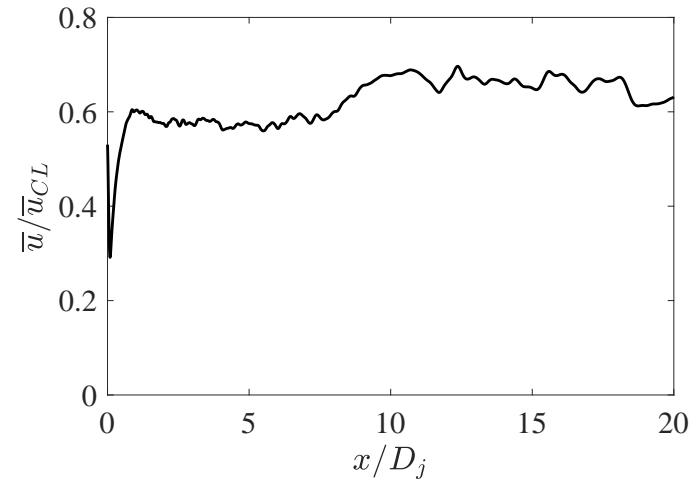

(b)

Fig. 5 Plots of axial velocity along a) SPS and jet centerline, normalized by jet velocity; and b) SPS, normalized by centerline velocity.

Figure $5 \mathrm{a}$ plots distributions of mean axial velocity on the jet centerline and on the SPS. The end of the potential core, defined here as the point where the centerline mean axial velocity $\bar{u}_{\mathrm{CL}}$ decays to $\bar{u}_{\mathrm{CL}}=0.95 U_{j}$, is located near $x=7.4 D_{j}$. The velocity on the SPS experiences a localized dip in the thin shear layer near the nozzle lip (likely a numerical artifact) and then maintains a fairly constant value at $\bar{u}=0.6 U_{j}$ up to $x=10 D_{j}$. Beyond this station it decays similarly to the decay of the mean centerline velocity. The value $U_{c} \approx 0.6 U_{j}$ is fairly well established in the shear-layer region of cold jets [38]. Previous works have shown that the mean axial velocity on the SPS can be used to model the convective velocity on that surface with reasonable accuracy [10, 11, 37]. Given the similarity between the curves shown in Fig. 57, it is interesting to see the resulting trend if we choose to normalize the velocity on the SPS by the centerline velocity at each axial location. The result is plotted in Fig. 5 b. As expected, the curve is nearly constant at $\bar{u}=0.6 \bar{u}_{\mathrm{CL}}$ for the extent 
of the potential core, after which the curve raises to approximately $\bar{u}=0.66 \bar{u}_{\mathrm{CL}}$ and remains roughly constant.

The momentum thickness of the flow is relevant to scaling relations that follow. To maintain the same definition within and downstream of the potential core, a generalization of the shear-layer momentum thickness is used:

$$
\delta_{\theta}=\int_{0}^{\infty} \frac{\bar{\rho}(r) \bar{u}(r)}{\bar{\rho}_{\mathrm{CL}} \bar{u}_{\mathrm{CL}}}\left(1-\frac{\bar{u}(r)}{\bar{u}_{\mathrm{CL}}}\right) d r
$$

where the centerline mean conditions are used for normalization. The growth $\delta_{\theta}(x)$, plotted in Fig. 6 is well captured by the linear fit $\delta_{\theta} / D_{j}=0.0226 x / D_{j}+0.0077$ throughout the region of interest. This result is in line with past work on this jet [18].

The radial location of the inner edge of the shear layer $r_{i}(x)$ is also relevant in scaling relations that will be developed. Within the extent of the potenial core, the inner edge is defined as the interior locus where the mean vorticity equals $0.05 U_{j} / D_{j}$, that is,

$$
\left|\bar{\omega}\left(x, r=r_{i}\right)\right|=0.05 \frac{U_{j}}{D_{j}}, r<D_{j} / 2
$$

Past the potential core, where the annular shear layer has collapsed on itself, we set $r_{i}=0$. The axial evolution of $r_{i}$ is plotted in Fig. 6 .

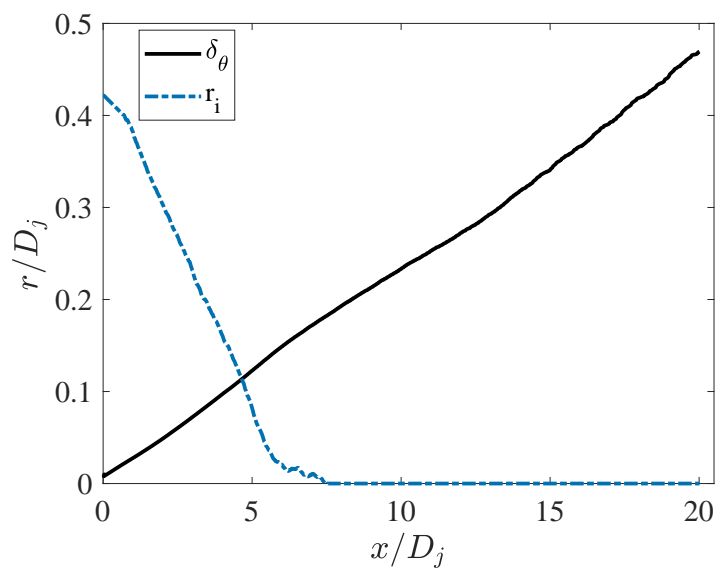

Fig. 6 Axial evolution of momentum thickness $\delta_{\theta} / D_{j}$ (solid black line) and inner bound of shear layer $r_{i} / D_{j}$ (dashed blue line).

Figure 7 plots isocontours of the normalized Reynolds stress magnitude $g / U_{j}^{2}$. The distribution of $g / U_{j}^{2}$ creates two distinct lobes that reach a minimum separation around $x / D_{j}=9$. Per its definition, the SPS is located along the peak of the Reynolds stress, resulting in a surface of approximately constant radius until $x / D_{j}=11$, where it starts diverging at a nearly constant rate of $4^{\circ}$ with respect to the jet axis. To quantify Fig. 77, we plot $g / U_{j}^{2}$ along the SPS in Fig. 8. The curve reaches a maximum value of $g / U_{j}^{2}=0.00894$ near $x / D_{j}=5$. For $2.15<x / D_{j}<7.5$ the Reynolds stress is nearly constant and close to the peak value, and then decays with downstream distance.

The statistics presented in Figures 4,8 were relevant to the SPS but not directly connected to the radiator surface. Next we present distributions of quantities that relate to the definition and physics associated with the radiator surface. Figure 9 plots the $x-r$ distribution of the convective velocity $U_{c_{p}}$ calculated from space-time correlations of the pressure fluctuation. Two distinct regions are noted: an inner region, encompassing the jet flow, where $U_{c_{p}}$ generally follows the trend of the mean axial velocity and is a fraction of the mean axial velocity (and is thus subsonic for this jet); and an outer region, near the edge of the jet and beyond, where $U_{c_{p}}$ 


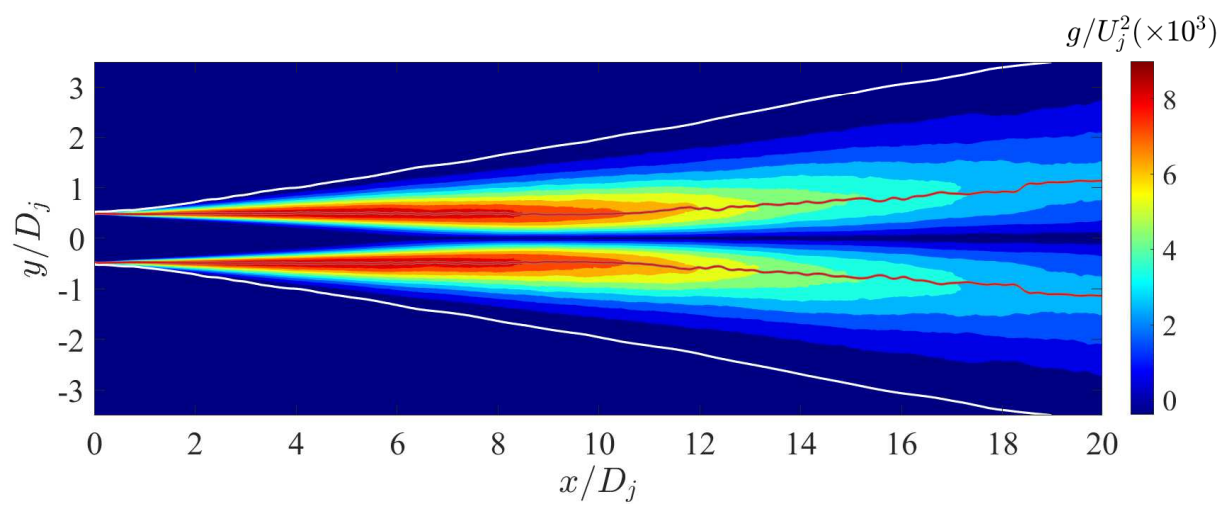

Fig. 7 Isocontours of normalized Reynolds stress magnitude $g / U_{j}^{2}$. Red line: SPS. White line: radiator surface.

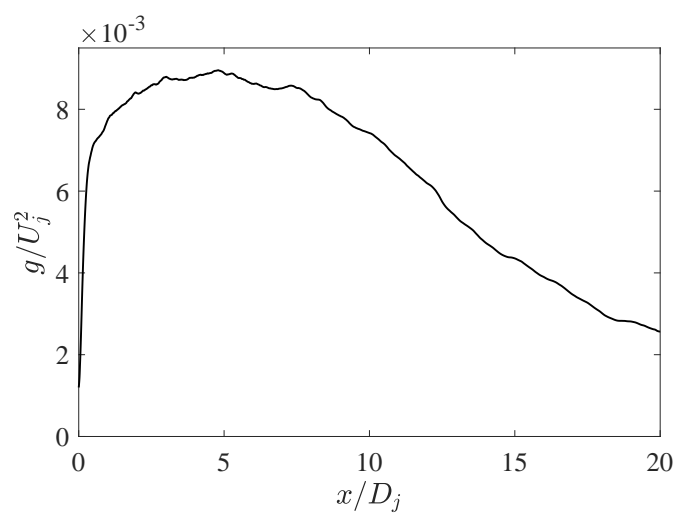

Fig. 8 Normalized Reynolds stress $g / U_{j}^{2}$.

rapidly increases to supersonic values indicative of the acoustic field (the speed of sound here equals $1.11 U_{j}$ ). Specifically, at a given axial location, $U_{c_{p}}$ starts from a peak value at the centerline and reduces radially as it follows the decline of the mean axial velocity; at some point near the edge of the jet, this trend reverses and $U_{c_{p}}$ starts increasing rapidly with radial distance. The radiator surface, defined as the surface where $U_{c_{p}}$ matches $U_{c_{u}}$ on the SPS, is located slightly outward of the position of this reversal, thus demarcates the transition from hydrodynamic to acoustic field. It should be noted that the region of supersonic convective velocity $U_{c_{p}}>1.11 U_{j}$ visible in the upper left of Fig. 9 does not indicate that pressure fluctuations travel at supersonic speeds, but rather that they propagate at large angles with respect to the horizontal displacements $\xi$ used for the computation of $U_{c_{p}}$. The convective velocity based on axial separation thus measures the trace of acoustic propagation in the axial direction.

Figure 10 a presents the distributions of convective velocity based on axial velocity $U_{c_{u}}$, and pressure fluctuation $U_{c_{p}}$ along the SPS. The two curves are almost identical, indicating that the turbulence scales imprint consistent velocity scales on pressure and velocity fluctuations. As mentioned above, previous works have surmised that the convective velocity can be modeled by the mean axial velocity along the SPS [37]. We test this hypothesis by comparing the curves of $U_{c}$ to that of $\bar{u}$ along the SPS, also shown in Fig. 10a. The curves are very similar qualitatively and only exhibit a small difference in values, with $U_{c_{u}}$ being about $8 \%$ higher than $\bar{u}$ for $x / D_{j}<10$.

The information presented in the contour plots of $U_{c_{p}}$ (Fig. 9) can be further quantified by presenting the radial distribution of $U_{c_{p}}$ at an axial station within the potential core, $x / D_{j}=5$. It is plotted in Fig. 


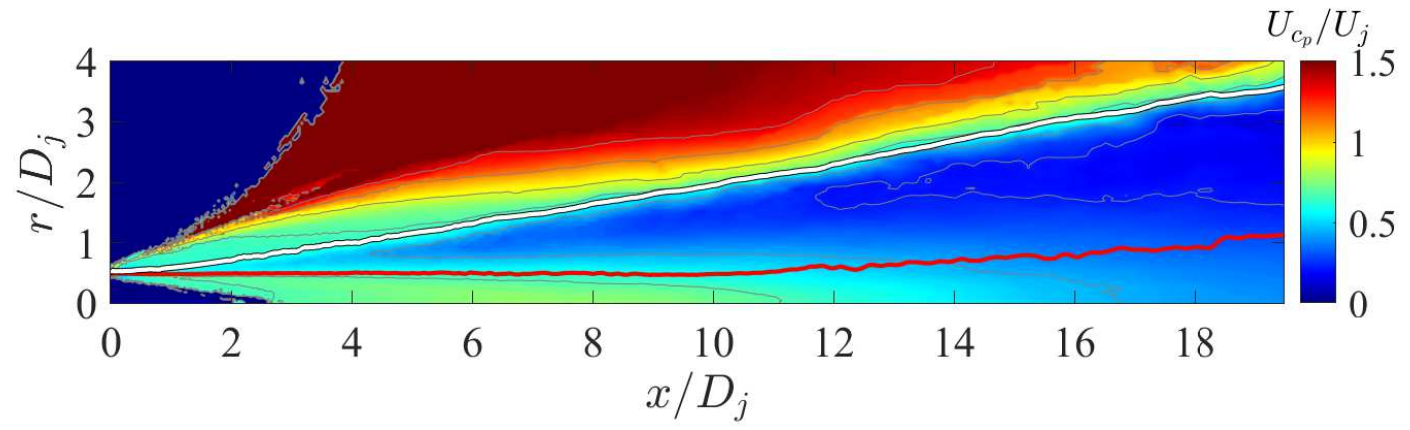

Fig. 9 Isocontours of normalized convective velocity $U_{c_{p}} / U_{j}$. Red line: SPS. White line: radiator surface.

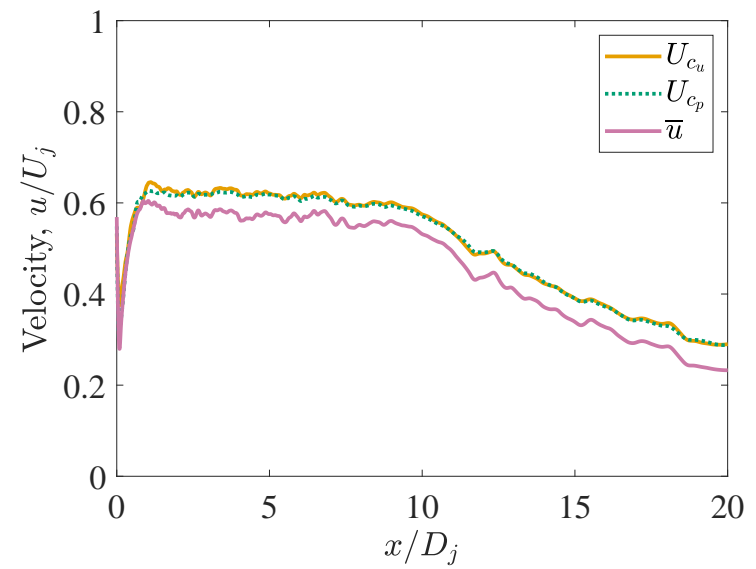

(a)

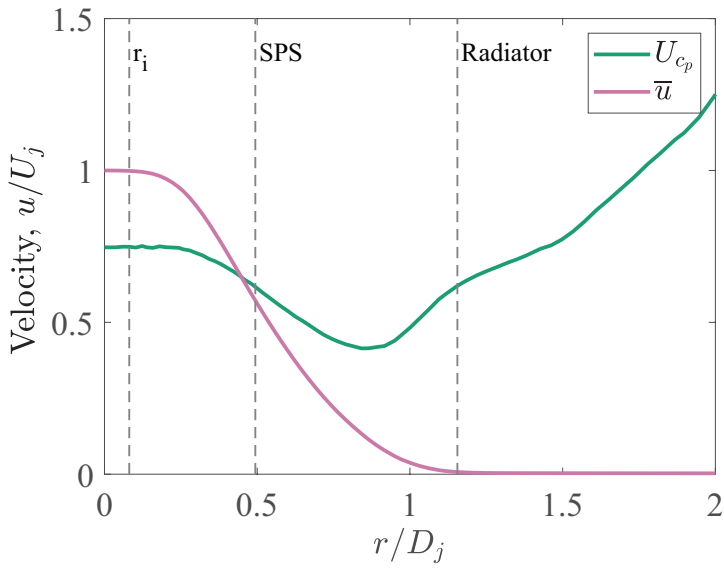

(b)

Fig. 10 Plots of convective velocity and time-averaged axial velocity a) along SPS; and b) radial trends at $x / D_{j}=5$.

$10 \mathrm{p}$, together with the variation of mean axial velocity velocity $\bar{u}$ at the same station. The potential core is manifested by the flat regions of the profiles near $r=0$. While $\bar{u}$ decays monotonically for $r>r_{i}, U_{c_{p}}$ first follows the trend of $\bar{u}$, reaches a minimum value near $\bar{u} / U_{j}=0.1$ (i.e., well within the vortical field), then reverses trend and increases rapidly as the hydrodynamic field transitions to acoustic. This reversal has also been seen in earlier LES of a single-stream jet [39]. It is very relevant to the understanding and modeling of the noise source.

Figure 11 plots isocontours of the magnitude of normalized mean vorticity $|\omega| D_{j} / U_{j}$. It is observed that the radiator surface is located at the edge of the mean vorticity field, consistent with its desired property to mark the transition from hydrodynamic to acoustic field. Figure 11 suggests that a criterion based on the mean vorticity may be used to located the radiator surface relying solely on the RANS solution. Initial efforts in this regard have shown promise [11] but further refinements are needed. 


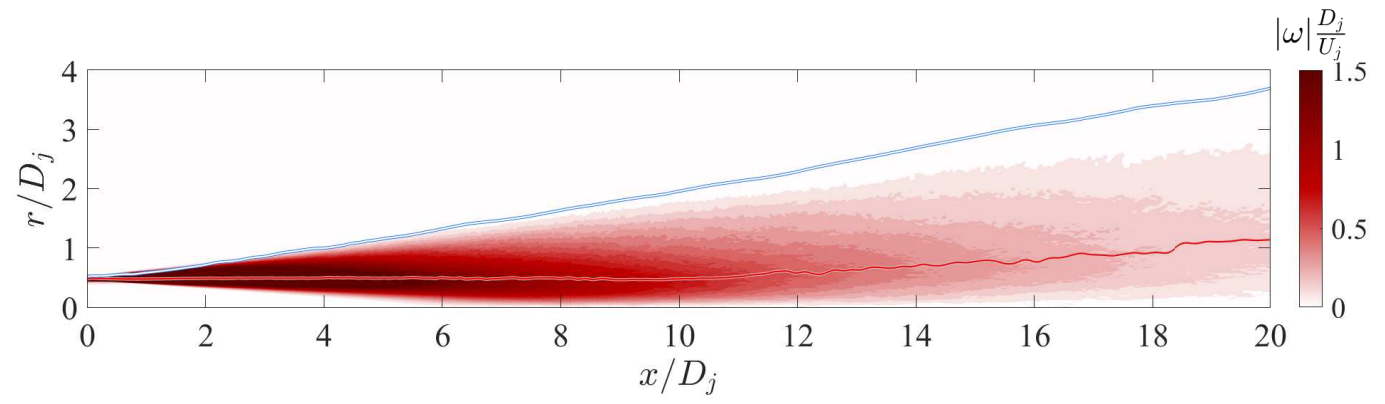

Fig. 11 Isocontours of normalized module of mean vorticity $|\omega| D_{j} / U_{j}$. Red line: SPS. Blue line: radiator surface.

\section{Distribution of Flow Scales}

\section{A. Fixed-Frame Length Scales}

We present the distributions of fixed-frame length scales as defined in Section III Isocontours of normalized length scale based on axial velocity fluctuation $L_{u} / D_{j}$ are plotted in Fig. 12. The value ranges from near-zero at the nozzle exit to a peak of $1.08 D_{j}$ inside the vortical field. In accordance to the expectations for its axial trend, the scales increase with the downstream distance as the jet turbulence structures are larger. In addition, the radial dependence shows some interesting trends. At a given axial location past the end of the potential core, $L_{u}$ has a radial trend whereby it increases outside the the centerline, reaches a local maximum within the jet, then decreases to a local minimum at the edge of the jet. Outside the jet, $L_{u}$ increases to very high values and saturates the contour. Upstream of the end of the potential core, $L_{u}$ exhibits a similar trend but starting at the inner edge of the shear layer instead of the jet centerline. We note that the local radial minimum of $L_{u}$ follows closely the radiator surface. This suggests a loss of coherence of velocity-based events near the edge of the jet. Near the SPS, $L_{u}$ experiences the strongest radial variation within the jet flow.

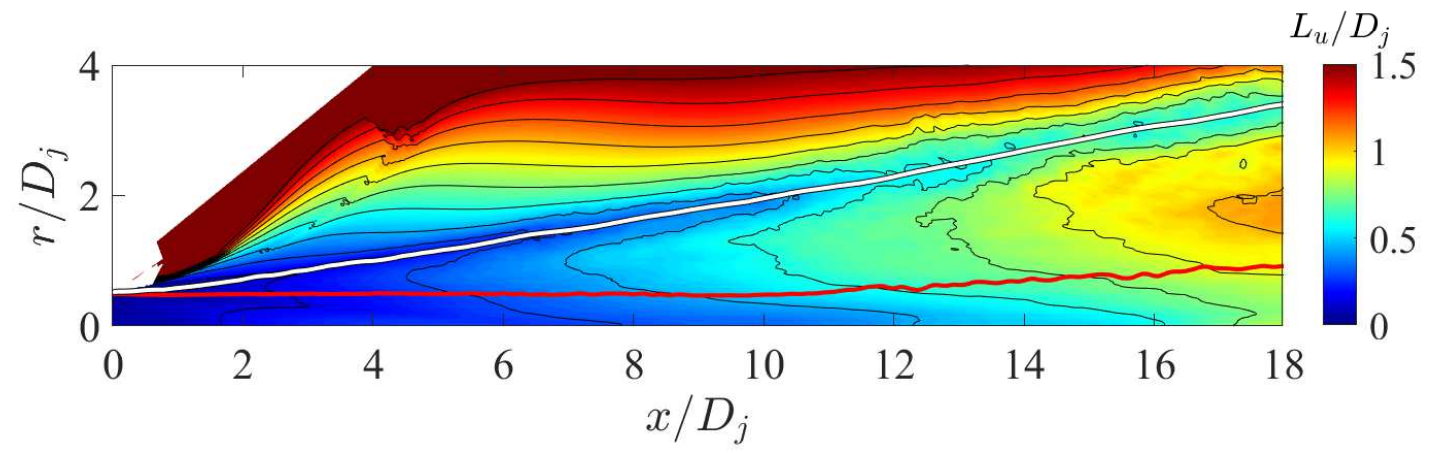

Fig. 12 Isocontours of normalized length scale $L_{u} / D_{j}$ based on axial velocity fluctuation. Red line: SPS. White line: radiator surface.

The distribution of normalized length scale based on pressure fluctuation, $L_{p} / D_{j}$, is shown in Fig. 13 . There are notable differences between velocity and pressure-based results. In this case, there is no local maximum inside the jet in the radial direction. Instead, the scales increase radially at a much higher gradient 
than in the axial direction, reaching a maximum around one nozzle diameter outwards from the radiator surface. Near the radiator surface, $L_{p}$ experiences its maximum radial gradient.

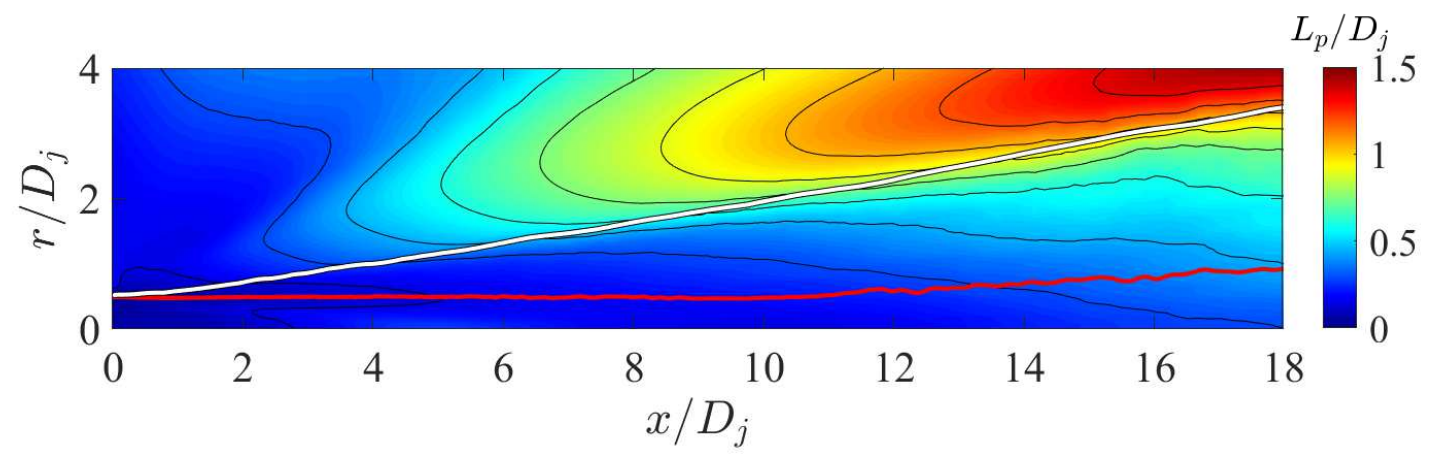

Fig. 13 Isocontours of normalized length scale $L_{p} / D_{j}$ based on pressure fluctuation. Red line: SPS. White line: radiator surface.

We interpret the radial trends of Figs. 12 and 13 as follows. The inner vortical region of the jet, marked by the SPS, contains the main events of mixing and momentum transport of the mixing layer (see Eq. 14). That region captures the largest scales of turbulence and by energy cascading it is also the location of small scales. That combination of large and small scales drives the value of the length scale $L$ below the actual length of the large scales [13]. As one leaves the SPS towards higher radial locations with lower vorticity, scales change to reflect the footprint of the turbulence in the inner region. There, the effects of small scale turbulence are lost faster than those of large scales, and less local influence of small eddies results in the increase of scales versus radius seen in Figs. 12 and 13 It appears that the pressure-based scales on Fig. 13 increase radially due to this effect until peaking beyond the radiator surface, well into the linear field. That region of radial maxima is due to the footprint of large scales vortical structures inside the jet [40, 41] that are not isolated by two-point correlations performed inside the vortical field. Velocity-based scales have a different behavior, whereby they capture better the extent of the eddies themselves but lose their footprint away from them. As one approaches the edge from the inside of the jet, velocity fluctuations disassociate from the inner vortical region and become affected by a combination of acoustic effects, sparse spurs of energy from inner regions, and small eddies of secondary acoustic relevance. This complex combination of influences causes very low velocity coherence and the "valley" of low length scale $L_{u}$ at the edge of the jet in Fig. 12. Finally, further out from the jet edge, the acoustic perturbations start to dominate and cause the increase in length scale in the near acoustic region.

The radial evolution of the flow length scales at eight axial stations ranging from $x / D_{j}=1$ to $x / D_{j}=15$ is plotted in Fig. 14p for $L_{u}$ and Fig. 14p for $L_{p}$. The radial trends appear to scale up with the downstream distance, so they may exhibit a type of self-similar behavior with the growth of the jet. To verify that hypothesis, we plot the radial distributions of $L_{u}$ and $L_{p}$ in Figs. 15 and 15 , respectively, at several axial positions and normalized by the momentum thickness $\delta_{\theta}$ of their corresponding location. Additionally, for the region surrounding the potential core, we define the origin of the radial trends as the inner edge of the shear layer, $r_{i}(x)$, as shown in Fig. 6 . Consequently, we plot the scales against $\left(r-r_{i}(x) / \delta_{\theta}(x)\right)$.

Figure 15 shows a fair collapse of the curves, particularly for axial locations at $x / D_{j} \geq 3$ for $L_{u}$ and $x / D_{j} \geq 5$ for $L_{p}$. Length scales at $x / D_{j}=1$ and $x / D_{j}=3$ acquire high values at low $r$, around $\left(r-r_{i}\right) / \delta_{\theta} \leq 3$, possibly indicating the influence of coherent waves within the potential core. Overall, all axial stations show common trends between the SPS (marked in black squares) and the radiator surface (blue 


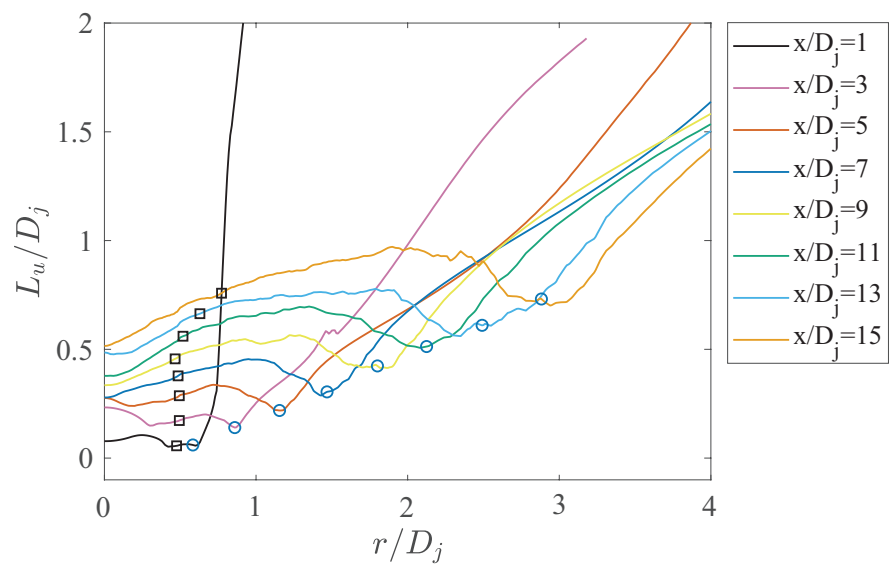

(a)

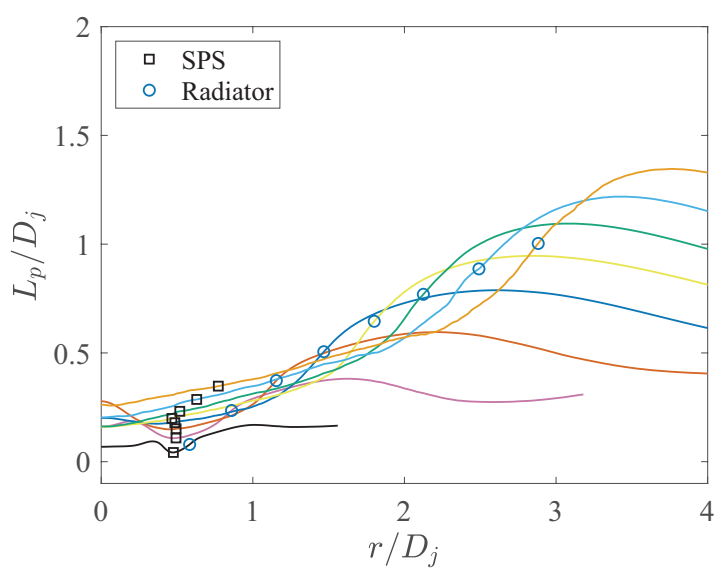

(b)

Fig. 14 Plots of length scale $L / D_{j}$ versus normalized radius $r / D_{j}$. a) Based on axial velocity. b) Based on pressure. Legend is the same in both figures.

circles) even though these trends differ between $L_{u}$ and $L_{p}$. At high radii of $\left(r-r_{i}\right) / \delta_{\theta} \geq 12$, the curves diverge as a result of the acoustic propagation at a variety of directions. Figure 15 is in agreement with the results by Proença et al. [14], by which fixed-frame $u^{\prime}$-based length scales of turbulence grow in accordance to the jet expansion along the centerline (past the potential core) and lip line. That conclusion is expanded here for the complete radial trend of the scales inside the vortical field, for both velocity and pressure-based scales, and axial positions starting from near the nozzle exit.

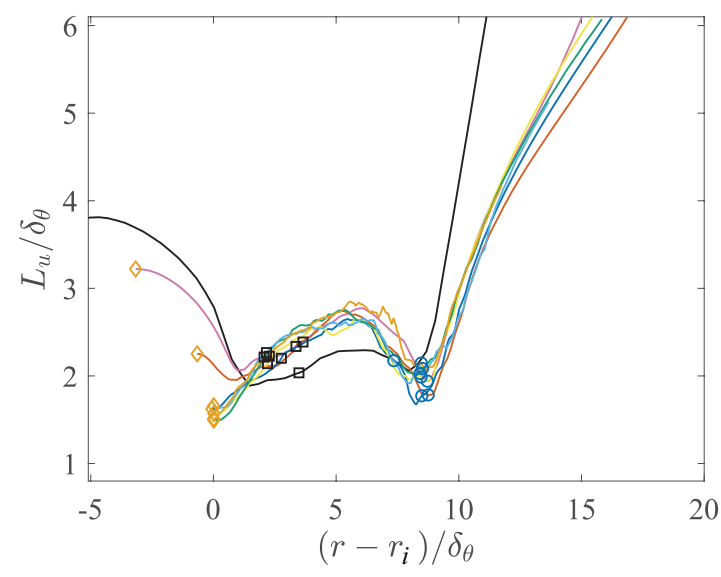

(a)

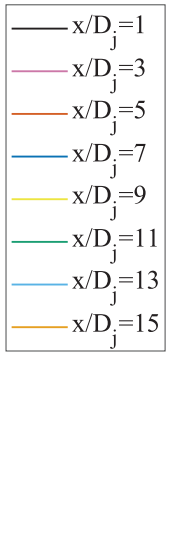

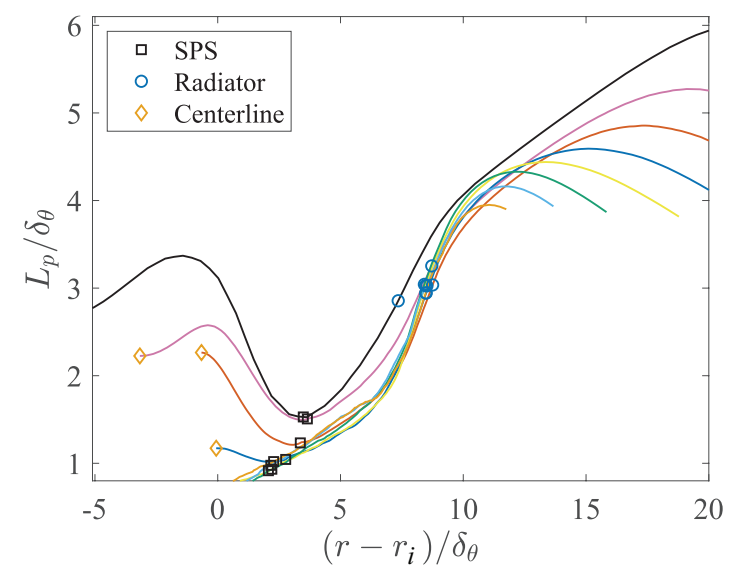

(b)

Fig. 15 Plots of normalized length scale $L / \delta_{\theta}$ versus normalized radius $\left(r-r_{i}\right) / \delta_{\theta}$. a) Based on axial velocity. b) Based on pressure. Legend is the same in both figures.

To quantify the connection between events on the SPS and radiator surface, Fig. 16a plots the the axial distribution of length scales $L_{u}$ and $L_{p}$ along these surfaces. All the curves exhibit a substantially linear growth along the first 15 jet diameters. In addition, the length scale on the SPS acquires a higher value when calculated from velocity fluctuation than from pressure fluctuation. This is an interesting result that challenges the intuitive prediction that velocity scales would be lower due to a richer content of high frequencies, and therefore smaller scales [40]. This relation reverses on the radiator surface, where the length scale is higher 
when based on pressure fluctuations. Comparing scales based on the same variable, it is evident that those on the radiator surface are consistently higher than those on the SPS, with the pressure-based scale exhibiting the strongest increase in this regard. These results suggest that the signature of eddies on the radiator surface is more coherent than the eddies themselves in the vortical field.

The most relevant connection to establish is between LES-based length scales is that of $L_{u}$ on the SPS, which would be more representative of the vortical field, and $L_{p}$ on the radiator surface, which would represent better the acoustic generation. However, this connection might need an adjustment such as a factor of proportionality. We proceed with that idea, and perform a simple linear fit of one to the other. The result is shown in Fig. 16b, where the distribution of $L_{p}$ on the radiator is adjusted to that of $L_{u}$ on the SPS by a factor of 0.74 . With the scaling the two curves practically collapse up to to $x / D_{j}=15$. This similarity is encouraging for a connection between the events on the two surfaces.

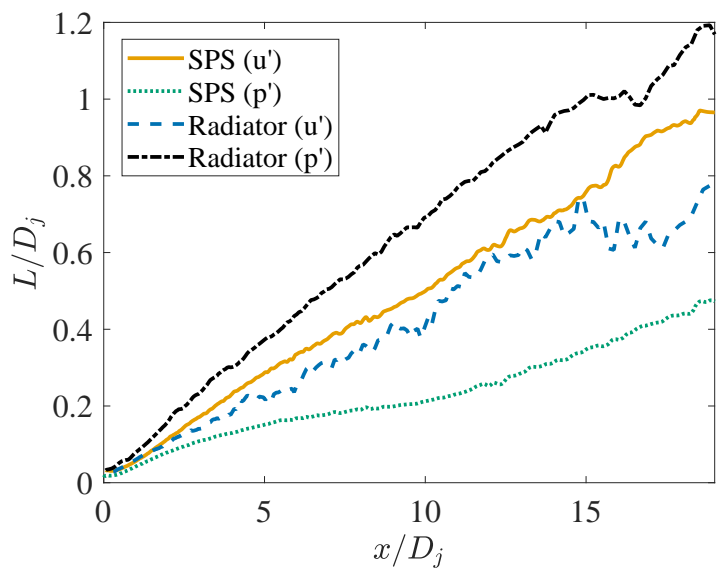

(a)

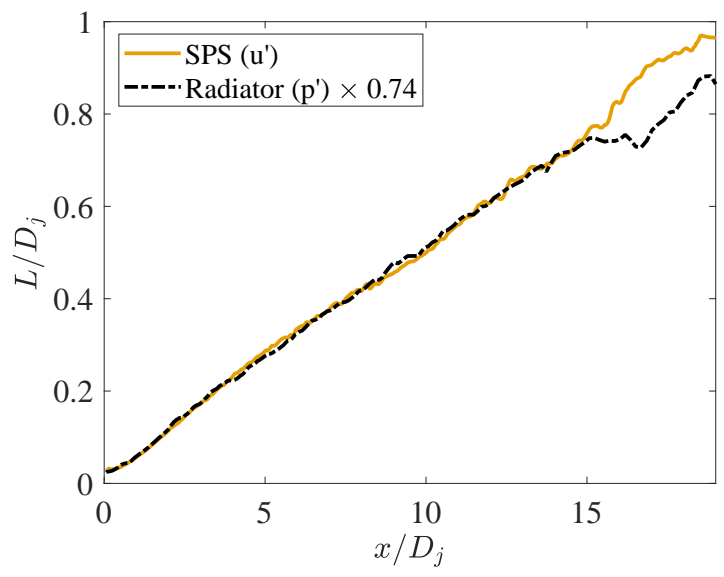

(b)

Fig. 16 Distribution of length scale $L$ on the relevant surfaces. Orange solid line: $L_{u}$ on SPS. Blue dashed line: $L_{u}$ on radiator surface. Green dotted line: $L_{p}$ on SPS. Black line dash-dot: $L_{p}$ on radiator surface. (a) Calculated values; b) Curve fitting between $L_{u}$ on SPS and $L_{p}$ on radiator surface, the latter is multiplied by a factor of 0.74 .

The next step in the analysis of fixed-frame length scales is the comparison with RANS-based (modeled) length scales. The latter need to be fitted to the LES-based scales with a factor of proportionality, as shown in Eq. 11. The result is presented in Fig. 17, where we plot the distribution of RANS-based length scale fitted over $L_{u}$ with a factor of $C_{L}=0.52$ along the SPS. The RANS-based length scale exhibits a linear growth similar to its LES-based counterpart, indicating the potential for RANS to provide meaningful information on the turbulence scale.

\section{B. Moving-Frame Length Scales}

The distribution of the normalized moving-frame length scale based on axial velocity fluctuation $\widehat{L}_{u} / D_{j}$ is presented in Fig. 18 The values range from near-zero at the nozzle exit plane to a peak of $\widehat{L}_{u} / D_{j}=3$ inside the vortical region. There is a strong dependence with axial and radial coordinates and some similarities with the fixed-frame length scales $L_{u}$. Again, $\widehat{L}_{u}$ increases with downstream distance and exhibits an interesting radial trend: starting from the jet centerline and going radially outwards, the values reach a local maximum within the jet and then decay to a local minimum at the edge of the jet. Outwards from the jet edge, the scales increase to very high values indicating strong coherence linked to acoustic propagation, although the quantitative values far outside the jet do not account for the angle of propagation. As with the fixed-frame 


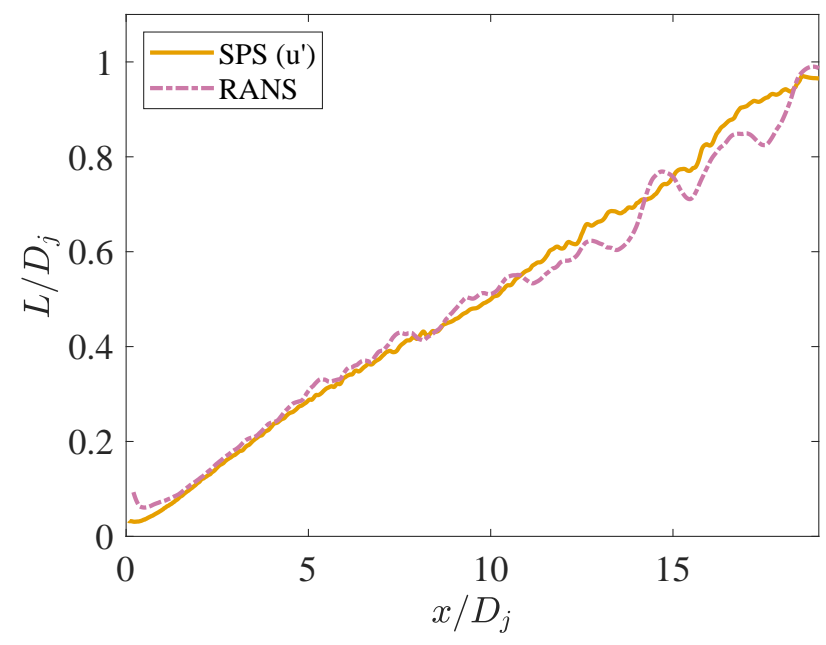

Fig. 17 Curve fitting of RANS-based length scale with LES-based length scale. Orange solid line: $L_{u}$ on SPS. Vermilion line dash-dot: RANS-based $L$ on SPS with $C_{L}=0.523$.

scale distribution of Fig. 12 , we note that $\widehat{L}_{u}$ experiences a minimum near the location of the radiator surface. On the other hand, the radial maxima of $\widehat{L}_{u}$ are more localized and occur inward relative to those of $L_{u}$, on the SPS.

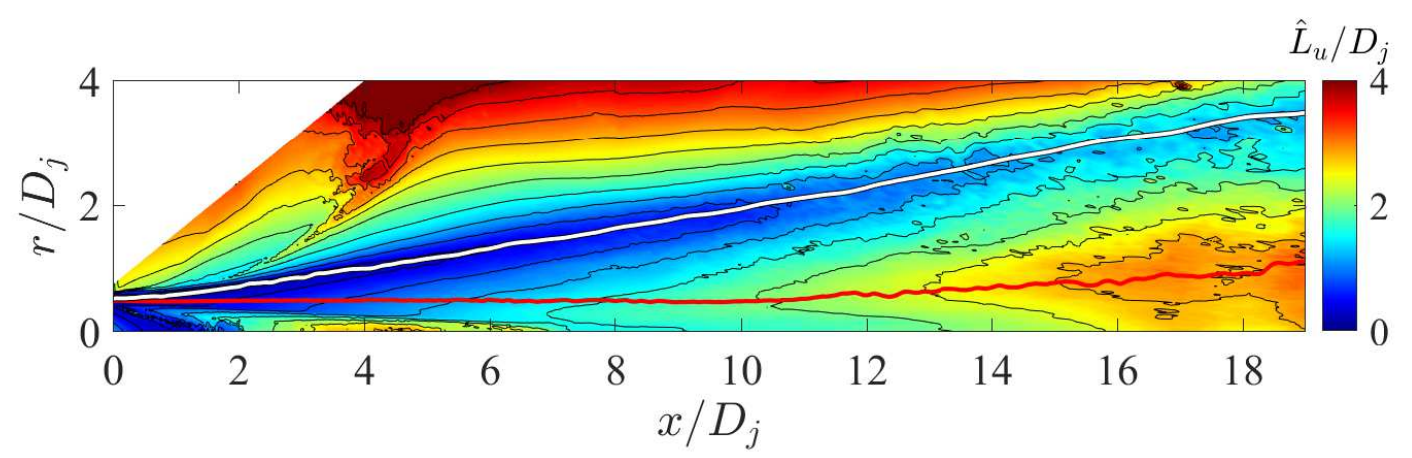

Fig. 18 Isocontours of normalized moving-frame length scale $\widehat{L}_{u} / D_{j}$ based on pressure fluctuation. Red line: SPS. White line: radiator surface.

The corresponding distribution of normalized moving-frame length scale based on pressure fluctuation, $\widehat{L}_{p} / D_{j}$, is shown in Fig. 19 . The values range from near-zero at the nozzle exit plane to $\widehat{L}_{p} / D_{j}=2$ within the vortical field. Within the radiator surface, $\widehat{L}_{p}$ follows the same general trends as $\widehat{L}_{u}$ but with lower values and a very weak maximum slightly outside the SPS. Immediately outside the radiator surface, $\widehat{L}_{p}$ rises rapidly to high values.

The radial trends of $\widehat{L}_{u}$ and $\widehat{L}_{p}$ are plotted in Fig. 20 using the same style as Fig. 15. The curves at locations past the end of the potential core tend to collapse well. Curves at early axial locations also show good collapse in the case of $\widehat{L}_{u}$ in Fig. 20a, but not in the case of $\widehat{L}_{p}$ in Fig. 20 p. It appears that, along the first diameters of the shear layer, the scale $\widehat{L}_{p}$ acquires larger values that what would correspond to the size of 


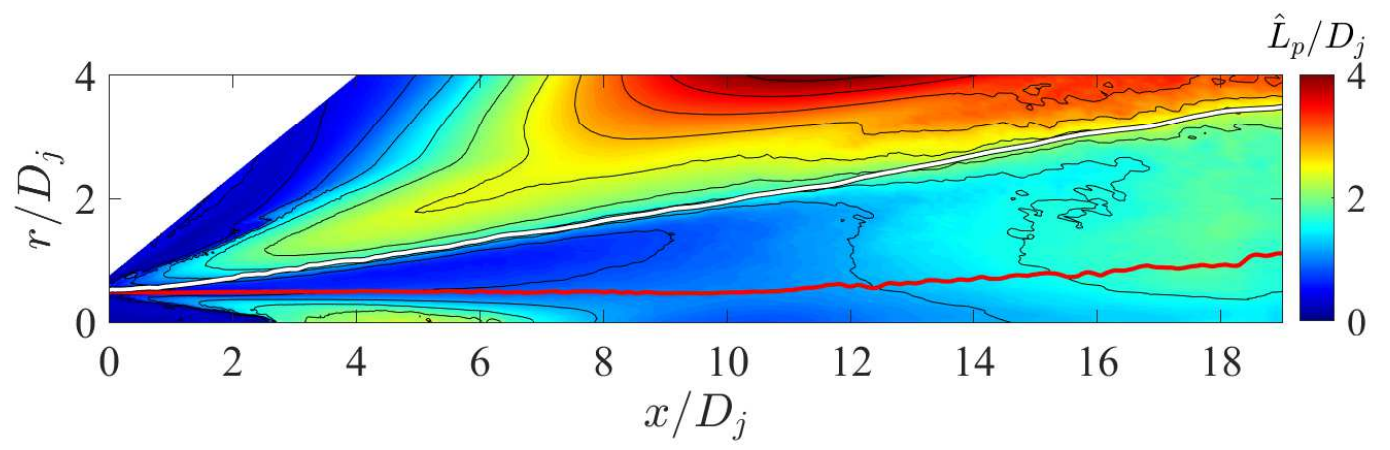

Fig. 19 Isocontours of normalized moving-fame length scale $\widehat{L}_{p} / D_{j}$ based on pressure fluctuation. Red line: SPS. White line: radiator surface.

the shear layer if it were self-similar. This is in contrast to the distribution of $\widehat{L}_{u}$, which is approximately self-similar and in agreement with the results by Proença et al. [14].

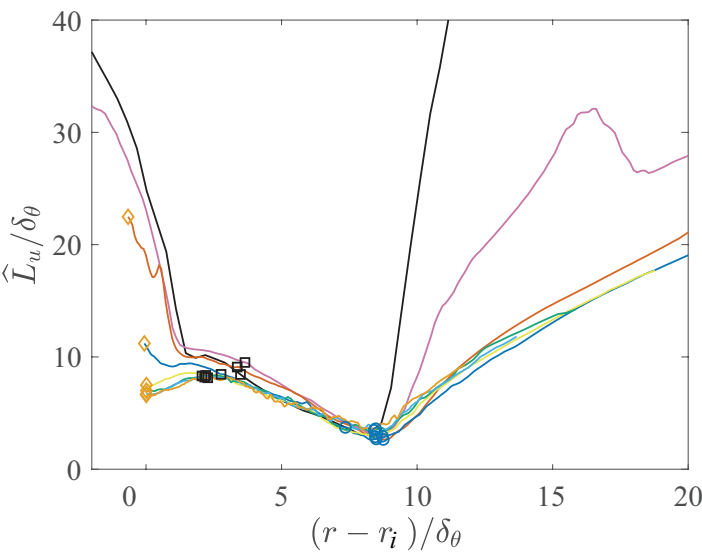

(a)

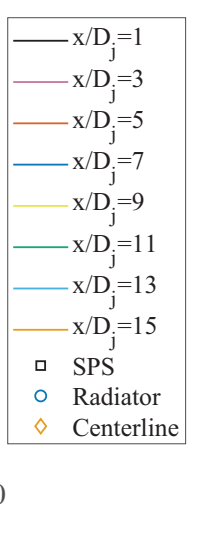

$\begin{array}{ll}\square & \text { SPS } \\ \circ & \text { Radiator } \\ \diamond & \text { Centerline }\end{array}$

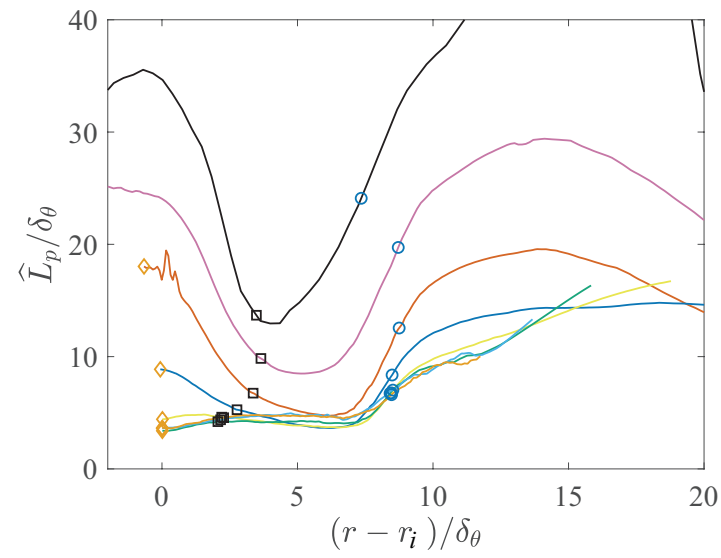

(b)

Fig. 20 Plots of normalized moving-frame length scale $\widehat{L}_{p} / \delta_{\theta}$ versus normalized radius $\left(r-r_{i}\right) / \delta_{\theta}$. a) Based on axial velocity. b) Based on pressure. Legend is the same in both figures.

The axial distributions of moving-frame length scales are plotted in Fig. 21 a. Contrary to the fixed-frame length scales, where all curves exhibit a linear growth, here only those based on axial velocity fluctuations increase linearly. The pressure-based length scale $\widehat{L}_{p}$ shows a rapid increase along the first few diameters of the jet, up to $x / D_{j} \approx 3$ on both SPS and radiator surface. $\widehat{L}_{p}$ acquires a local maximum near $x / D_{j}=4$ on the radiator surface, and starts growing in a roughly linear fashion after $x / D_{j}=7.5$, very near the end of the potential core. Similarly to the fixed-frame length scales, $\widehat{L}_{u}$ is higher than $\widehat{L}_{p}$ on the SPS, but lower on the radiator surface.

The non-linear growth of $\widehat{L}_{p}$ on the radiator surface makes it difficult to relate to the linearly-growing $\widehat{L}_{u}$ on the SPS. A simple, proportional curve fit that attempts to match the entire axial span of the curves presented in Fig. 21 a would show no apparent similarity. In fact, figure 20 p shows that the high values of $\widehat{L}_{p}$ on the first jet diameters are not limited to the radiator surface, but exist in the whole radial extent along the 
first diameters of the jet. Downstream of the potential core, the values of $\widehat{L}_{p}$ appear to be consistent with the linear jet growth. That observation is validated by the linear trend of $\widehat{L}_{p}$ in Fig. 21 after $x / D_{j}=7.4$. We can tentatively fit the curve of $\widehat{L}_{p}$ on the radiator surface to that of $\widehat{L}_{u}$ on the SPS, using only the data past the end of the potential core. The result is shown in Fig. $21 \mathrm{p}$ for a proportionality value of 1.22, where we observe good similarity along the fitted axial span. The high initial growth of $\widehat{L}_{p}$, and the fact that it forms a local maximum along the radiator, are interesting findings that remain under investigation.

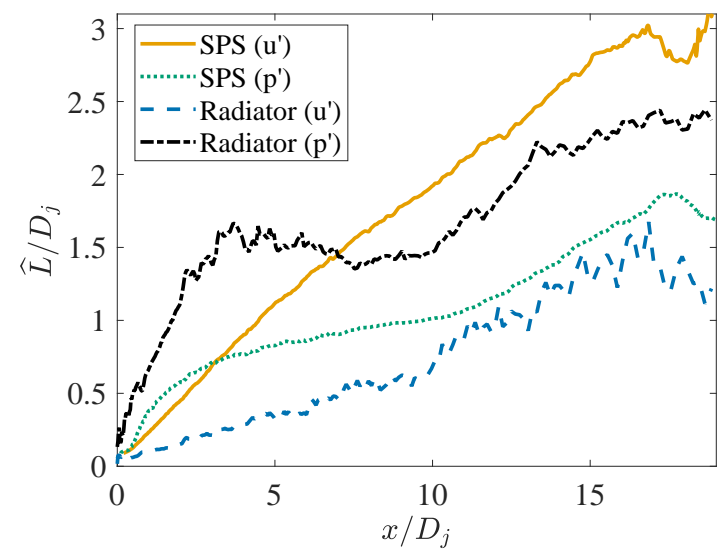

(a)

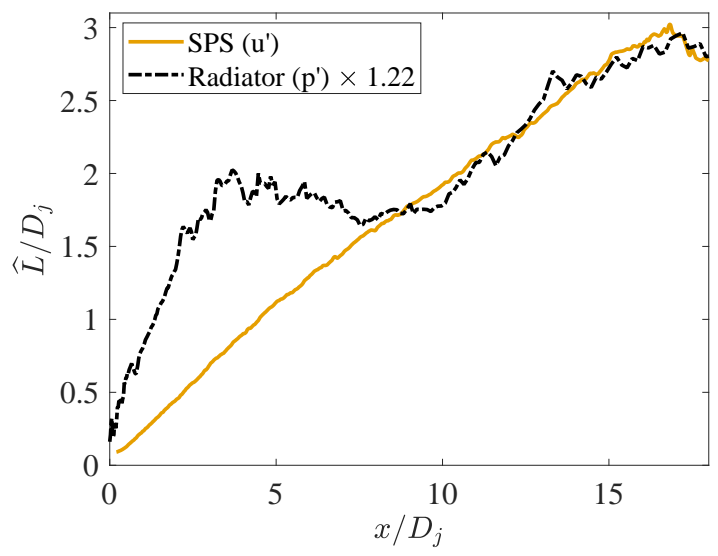

(b)

Fig. 21 Distribution of length scale $\widehat{L}$ on the relevant surfaces. Orange solid line: $\widehat{L}_{u}$ on SPS. Blue dashed line: $L_{u}$ on radiator surface. Green dotted line: $\widehat{L}_{p}$ on SPS. Black line dash-dot: $\widehat{L}_{p}$ on radiator surface. (a) Calculated values; b) Curve fitting of $\widehat{L}_{p}$ on radiator surface, multiplied by a factor of 1.22, to $\widehat{L}_{u}$ on SPS.

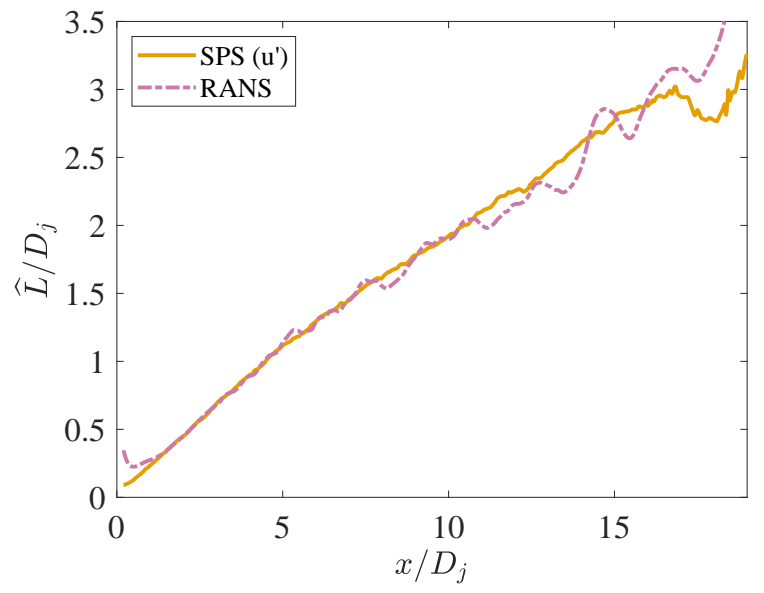

Fig. 22 Curve fitting of RANS-based length scale with LES-based moving-frame length scale. Orange solid line: $\widehat{L}_{u}$ on SPS. Vermilion line dash-dot: RANS-based $L$ on SPS with $C_{L}=1.94$.

After the analysis of LES-based scales $\widehat{L}$, we compare their evolutions with those of RANS-based length scales on the SPS. The result is plotted in Fig. 22. The curve of RANS-based length scales is fitted to the distribution of $\widehat{L}_{u}$ through a factor of $C_{L}=1.94$. Similarly to the case of fixed-frame length scales, both trends are linear and easily fitted with a proportionality factor. On the other hand, the distribution of pressure-based 
scales $\widehat{L}_{p}$ on the SPS does not have such a linear growth and would not render the same agreement when compared to RANS. Pressure-based scales would require more than a simple linear fit to the RANS scales, a topic that is under investigation.

\section{Azimuthal Scales}

We now present the distribution of azimuthal scales $\Phi$ calculated by two-point correlations as defined in section III Figures 23 and 24 plot isocontours of azimuthal scales based on axial velocity and pressure fluctuations, respectively, and include the locations of the SPS and radiator surface. Referring to Fig. 23, at low radius $r<0.2 D_{j}$ the azimuthal scale saturates to $\Phi_{u}=\pi$, indicating strong coherence. This is expected because events at $r \rightarrow 0$ are axisymmetric and correlated. With increasing radius, $\Phi_{u}$ drops rapidly to $\sim 0.12 \pi$ near the SPS and continues decaying until it reaches a radial minimum inside the jet. Continuing outwards, $\Phi_{u}$ increases rapidly outside the jet. The saturated portion of the contour plot suggests that $u^{\prime}$-based azimuthal correlations are not appropriate for characterizing the entirety of the acoustic field. The distribution of azimuthal scales based on pressure $\Phi_{p}$, displayed in Fig. 24, shows low values within the vortical field away from the centerline similar to the distribution of scales based on velocity, $\Phi_{u}$. Near the edge of the jet, $\Phi_{p}$ rises rapidly to form a trend of radial maxima outside the radiator surface. This rapid increase near the jet edge suggests a change in the nature of the disturbances: from compact turbulence eddies of limited azimuthal span inside the jet to an azimuthally coherent linear signal outside the jet.

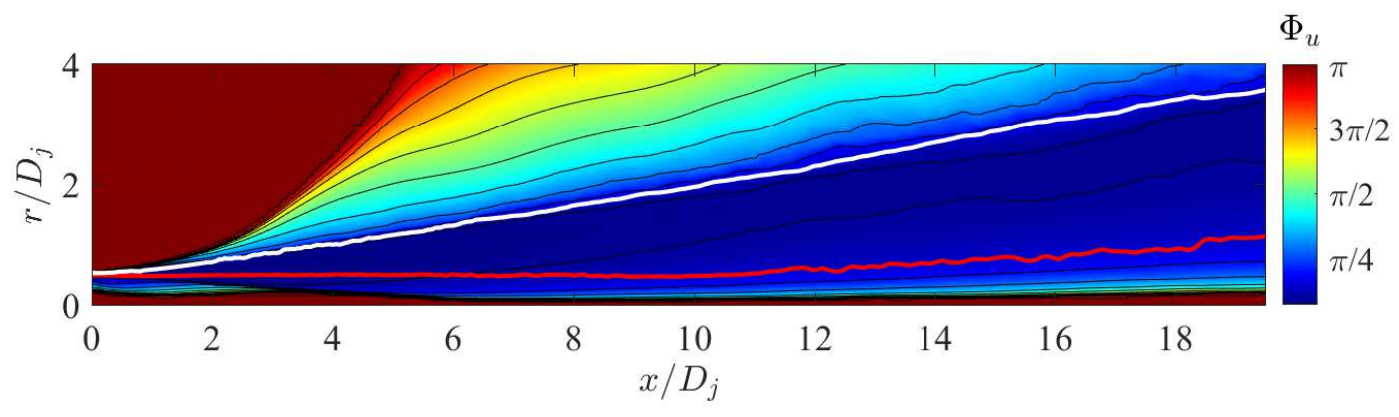

Fig. 23 Isocontours of azimuthal scale $\Phi_{u}$ based on axial velocity fluctuation, in radians. Red line: SPS. White line: radiator surface.

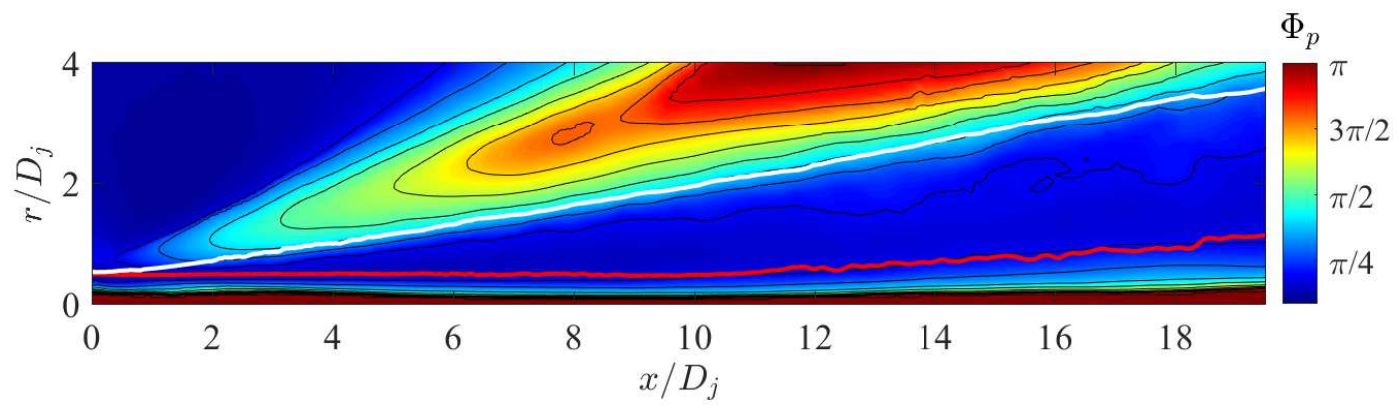

Fig. 24 Isocontours of azimuthal scale $\Phi_{p}$ based on pressure fluctuation, in radians. Red line: SPS. White line: radiator surface. 
We now study the radial trends of $\Phi_{u}$ and $\Phi_{u}$. As with the axial length scales, it is interesting to see if these trends are consistent with the jet expansion, quantified by the radial momentum thickness $\delta_{\theta}$. We plot the result in Fig. $25 \mathrm{a}$ for $\Phi_{u}$ and Fig. $25 \mathrm{p}$ for $\Phi_{p}$. The curves at various axial stations show very good collapse, particularly those at $x / D_{j} \geq 5$. Near the nozzle exit (represented by the curves of $x / D_{j}=1$ ) the radial trends are qualitatively, but not quantitatively, similar to the common trends at larger axial locations.

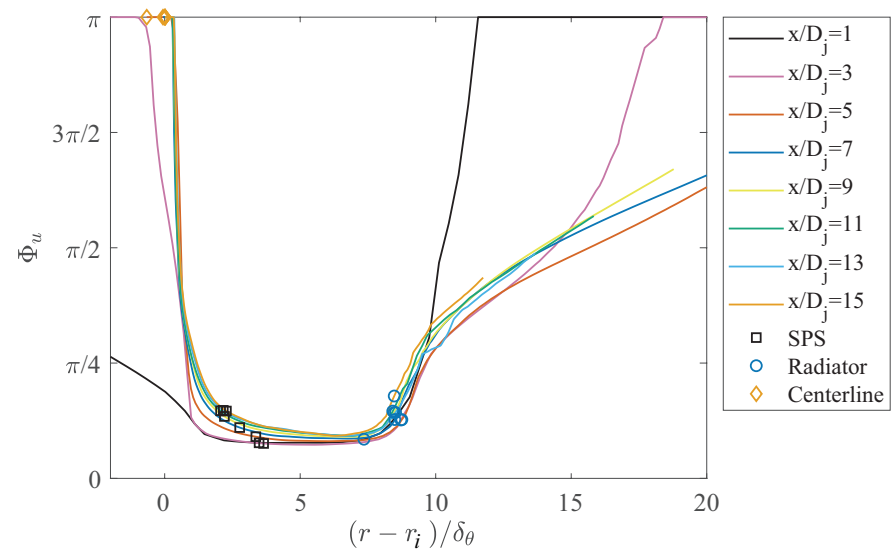

(a)

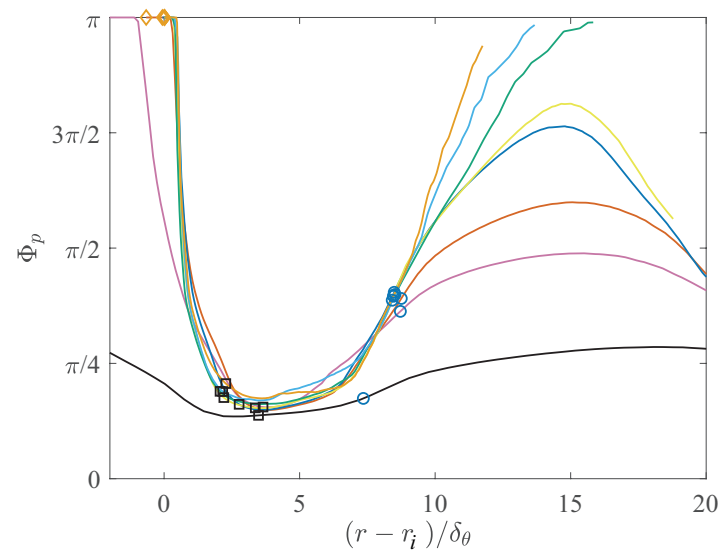

(b)

Fig. 25 Plots of azimuthal scales $\Phi$ versus normalized radius $\left(r-r_{i}\right) / \delta_{\theta}$. a) Based on axial velocity. b) Based on pressure. Color legend is the same in both figures.

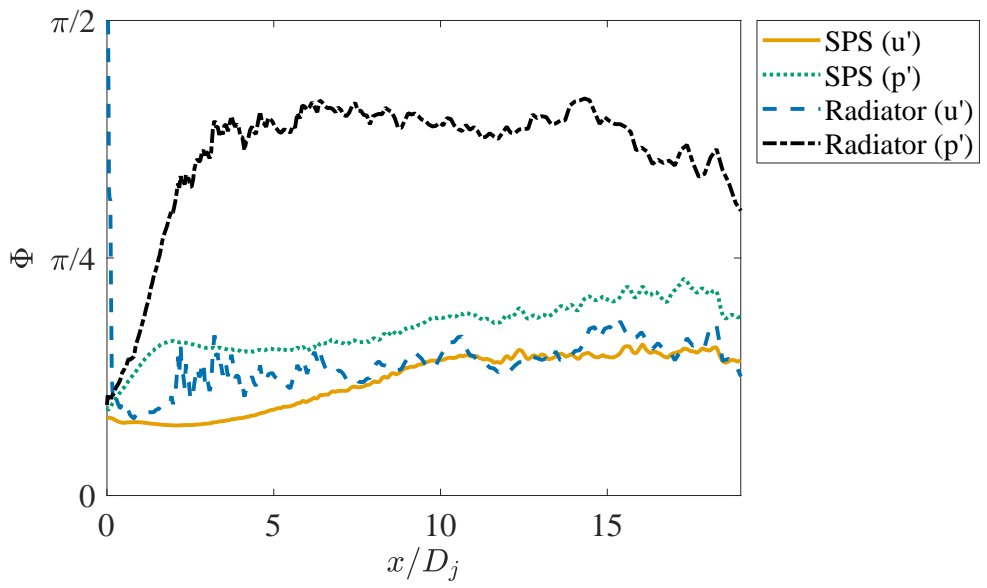

Fig. 26 Distribution of azimuthal scale $\Phi$ on the relevant surfaces. Orange solid line: $\Phi_{u}$ on SPS. Blue dashed line: $\Phi_{u}$ on radiator surface. Green dotted line: $\Phi_{p}$ on SPS. Black line dash-dot: $\Phi_{p}$ on radiator surface.

Axial distributions of $\Phi_{u}$ and $\Phi_{p}$ on the SPS and radiator surface are plotted in Fig. 26. All curves exhibit a slower growth than those of axial length scales shown earlier. The flatness of the azimuthal scales indicates that the azimuthal coherence of the turbulence eddies is not necessarily linked to their growth in the $(x, r)$ plane. The curves of $\Phi_{u}$ along the SPS and radiator, and $\Phi_{u}$ along the radiator, show a similar range of values between $\Phi=0.29$ and $\Phi=0.68$ approximately. The distribution of $\Phi_{p}$ along the radiator surface exhibits a notably different trend: it starts with a steep rise up to $\Phi_{p}=1.2$ at $x / D_{j}=3.3$, remains flat until $x / D_{j}=15$, then decreases slightly. The difference between $\Phi_{p}$ on the radiator surface and $\Phi_{u}$ on 
the SPS makes the connection between the two surfaces difficult, at least when only attempting a simple proportionality factor. Such attempt, which indeed yields qualitative differences between the trends, is plotted in Fig. 27a. This result indicates that there is no straight-forward, linear connection between integral values of azimuthal scales along the SPS and radiator. Further clarity may come from using frequency-resolved methods, modal analysis, and separation of hydrodynamic/acoustic fields to better understand the trends. The LES-based distribution of $\Phi_{u}$ is compared with the with RANS-based azimuthal scale (Eq. 12) in Fig. 27b, where the RANS-based distribution is fitted with a factor $C_{\Phi}=0.24$. There is a modest qualitative agreement between the two curves, particularly past $x / D_{j}=5$.

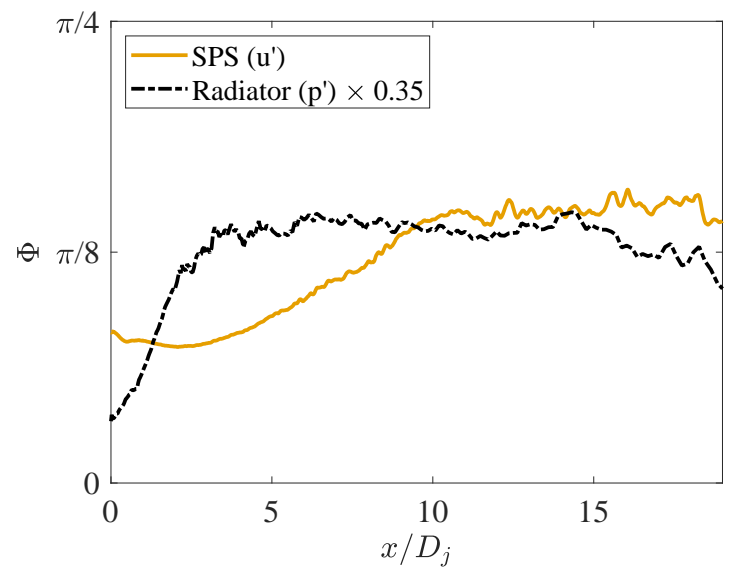

(a)

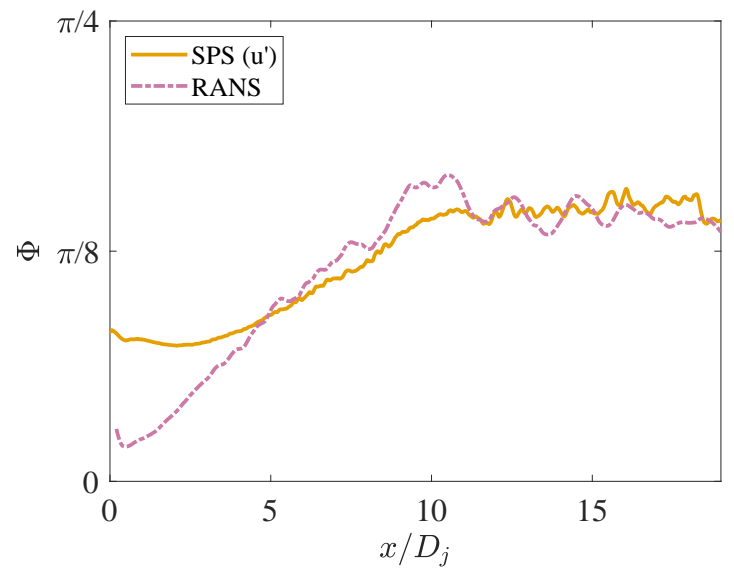

(b)

Fig. 27 Curve fitting of azimuthal scales: a) $0.35 \Phi_{p}$ on radiator surface to $\Phi_{u}$ on SPS; b) RANS-based $\Phi$ on the SPS, with $C_{\Phi}=0.24$, to $\Phi_{u}$ on the SPS.

\section{Two-Dimensional Correlations}

The physical mechanisms that cause the scale distributions discussed in sections VI.A and VI.B can be further investigated by studying the shape of their associated space-time correlations. Moreover, correlations performed on a longitudinal plane using a fixed reference point can give valuable insight of the physical phenomena at play [18]. In this section we present normalized correlations $R_{u и}$ and $R_{p p}$ for two reference locations on the SPS.

Space-time correlations $R_{u и}$ and $R_{u и}$ on an azimuthal plane are plotted in Fig. 28 for three time separations. The reference point is on the SPS at $x=3 D_{j}$. The normalized time separations are $\tau U_{j} / D_{j}=-1.94$ (top row), 0 (middle row) and 1.94 (bottom row). A vertical dashed line indicates the position that corresponds to a downstream convective velocity $U_{c}=0.6 U_{j}$. $R_{u и}$ shows a compact area of correlation, mostly limited to a small positive (red) region that travels along the SPS and a negative (blue) oblong region along the radiator surface. The oblong structure travels with $U_{c}<0.6 U_{j}$. On the other hand, $R_{p p}$ shows a wavy structure with strong radial correlation traveling fairly uniformly at a speed near $0.6 U_{j}$. An intriguing characteristic of the pressure-based structure is that the correlation at non-zero time delays exhibits peaks near the inner and outer edges of the shear layer. The difference in location, shape, and convective velocity of the features of $R_{u u}$ and $R_{p p}$ suggest that the two correlations are portraying different natures of events. The $u^{\prime}$-based correlations reflect compact turbulence events that travel along the SPS and leave their footprint at the edge of the jet in the form of a negative region of correlation. The $p^{\prime}$-based correlations appear sensitive to interactions of eddies with the potential flow around them. These observations may explain the difference between moving-frame length scales $\widehat{L}_{u}$ and $\widehat{L}_{p}$ of section VI.B and Fig. 21 . While $\widehat{L}_{u}$ follows the growth of the vortical region, 
$\widehat{L}_{p}$ appears affected by large-scale events that result from interactions of eddies with the potential flow surrounding them; thus, $\widehat{L}_{p}$ grows faster than the vortical region in the initial few diameters of the jet.

(a)

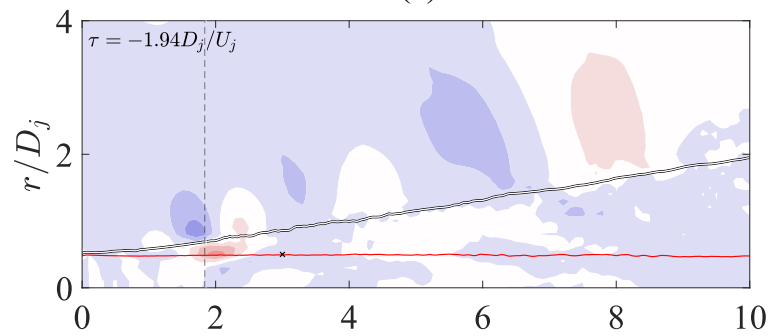

(c)

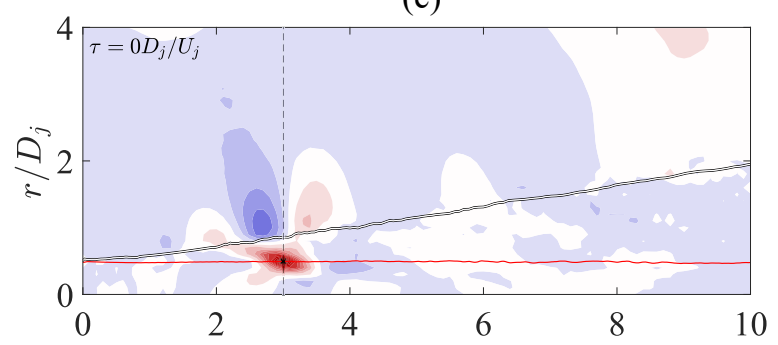

(e)

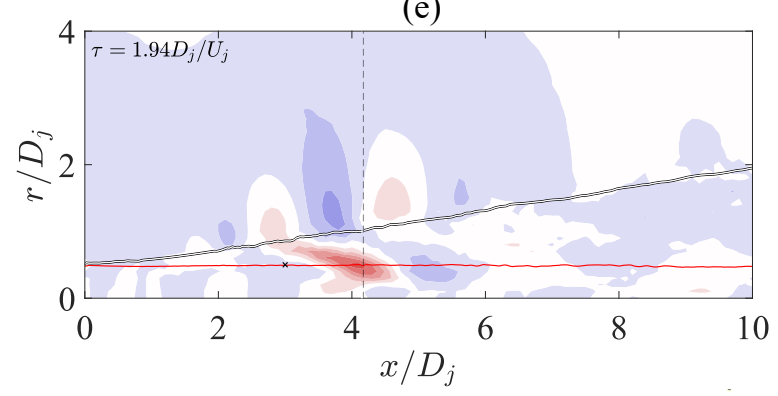

(b)

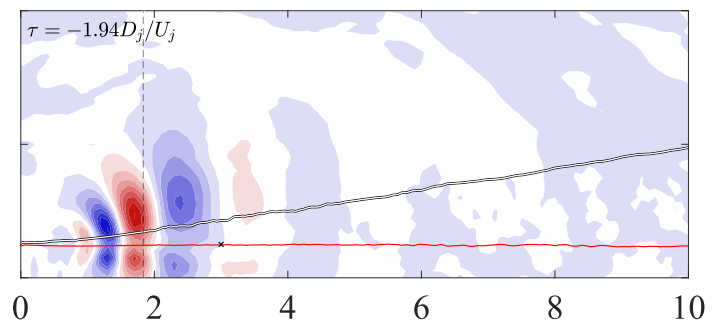

(d)

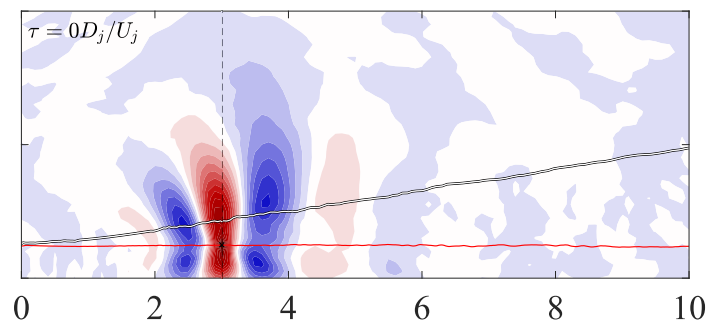

(f)

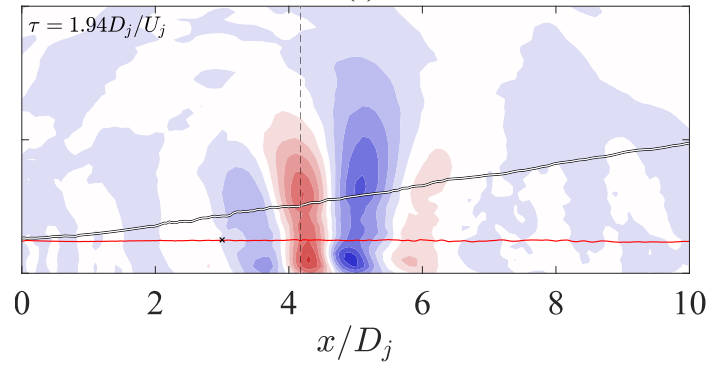

Fig. 28 Contours of $R_{u u}$ (left column) and $R_{p p}$ (right column) with reference point on SPS at $(x, r)=$ $(3,0.5) D_{j}$. Time separations: $\tau=-1.94 D_{j} / U_{j}$ (top row), $\tau=0$ (middle row), and $\tau=1.94 D_{j} / U_{j}$ (bottom row). Red line: SPS. White line: radiator surface. Black dashed vertical line: positions for a downstream convection at $0.6 U_{j}$. Color scale ranges from $\mathbf{- 0 . 7 5}$ (blue) to $+\mathbf{0 . 7 5}$ (red).

We now study the evolution of $R_{u u}$ and $R_{p p}$ with the reference point on the SPS at $x=7.5 D_{j}$, very near the axial location of the end of the potential core. Figure 29 displays the resulting correlations at time separations $\tau U_{j} / D_{j}=-3.49$ (top row), 0 (middle row) and 3.49 (bottom row). The time progression of $R_{u u}$ shows a compact structure of positive correlation traveling along the SPS; its effects are felt above the radiator surface as a region of negative correlation. At $\tau=0$, the peak of the negative correlation is -0.24 . The limited size of the positive structure indicates that the $u^{\prime}$ correlations capture the local but not global effects of the convecting eddies. The axial velocity fluctuation thus becomes decorrelated as we move from the SPS to the outer edge of the jet. This explains the region of low correlation between positive and negative structures in Fig. 29 (a,c,d), as well as the "valley" of $u^{\prime}$-based length scales in Fig. 12 for $L_{u}$ and Fig. 18 for $\widehat{L}_{u}$.

There is an additional feature of the contours of $R_{u u}$ of Fig. 29. The positive structure seems to rotate counter-clockwise as a result of the convective velocity decreasing with radius within the vortical region [11]. The gradient in convective velocity also explains the difference in the location of the radial maxima in the distributions of $L_{u}$ and $\widehat{L}_{u}$ (Figs. 12 and 18 , respectively). While the fixed-frame length scale $L_{u}$ peaks 
outwards from the SPS, the moving-frame length scale $\widehat{L}_{u}$ is extended by the higher convective velocity on the SPS and thus peaks on the SPS. This effect is visible in Fig. 29k, where the structure of positive correlation has traveled further downstream along the SPS than above it.

The time progression of $R_{p p}$ is seen in Fig. 29(b,d,f). A wavepacket-like structure is evident for all the time separations shown. At zero time separation, contour (d), a region of strong positive correlation emanates as a radial beam from the reference point to all across the jet, from the centerline to well outside the radiator surface. It is evident how the footprint of the large-scale structures is imprinted on the radiator surface. For negative and positive time separations, contours (b) and (f), similar but weaker structures are seen, with rapid decorrelation for $\tau>0$. The latter may be explained by the decline of the jet velocity past the end of the potential core and accompanying weakening of sound generation. It is notable that the $R_{p p}$ correlations structures convect fairly uniformly with a speed near $0.6 U_{j}$, while the $R_{u u}$ structures do not progress uniformly and their convective speed depends on radial location. These observations lend further credence to the idea reasoned earlier that pressure-based scales are influenced by the interaction of large eddies eddies with the surrounding potential flow and not by the local scales of turbulence. This also means that pressure-based scales may not capture local events as accurately as velocity-based scales. These observations may shed light on the dilemma noted in sections VI.A and VI.B. namely that higher values of $u^{\prime}$-based length scales than $p^{\prime}$-based scales on the SPS were challenging to interpret as the $p^{\prime}$ signal is expected to filter the short wavelength components present in $u^{\prime}$. In fact, vortex identification methods usually rely on velocity, not on pressure [42]. Velocity fluctuations describe better the shape of the turbulence eddies and remain correlated for longer distances than pressure fluctuations, but they do not reflect the footprint of the eddies inside the vortical field.

(a)

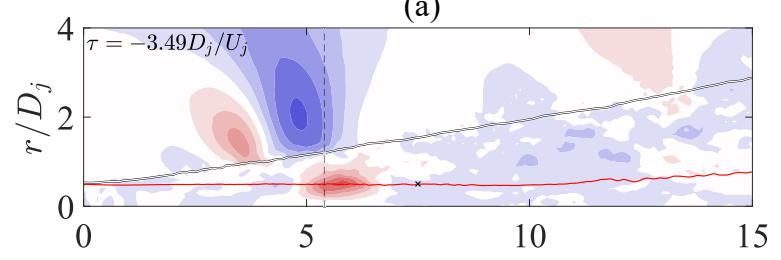

(c)

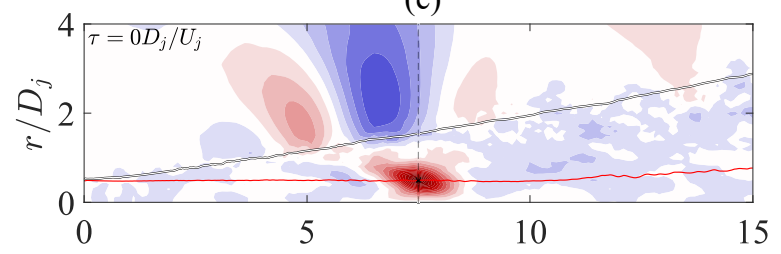

(e)

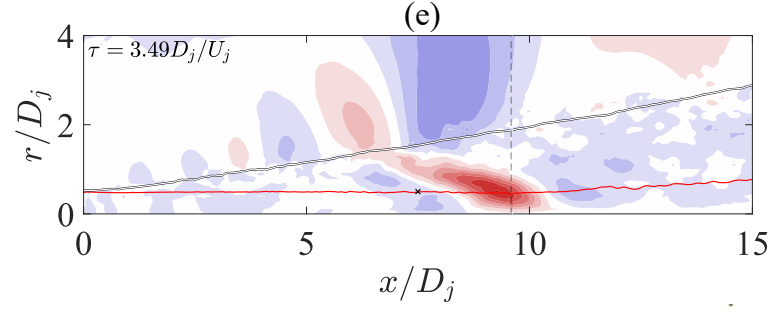

(b)

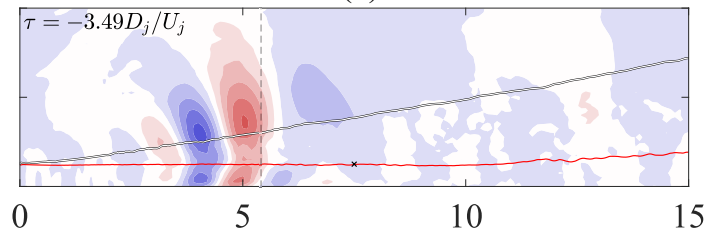

(d)

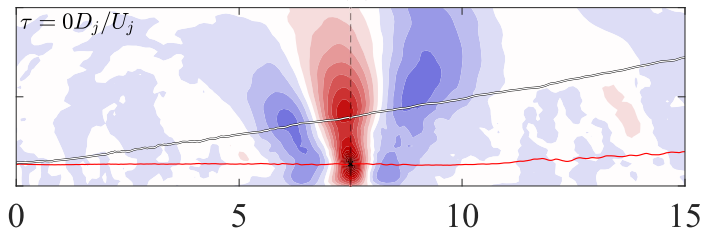

(f)

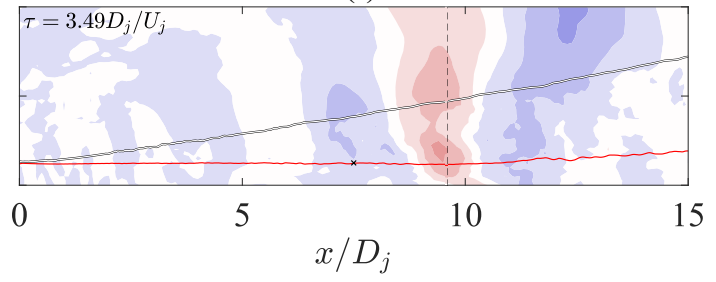

Fig. 29 Contours of $R_{u u}$ (left column) and $R_{p p}$ (right column) with reference point on SPS at $(x, r)=$ $(7.5,0.5) D_{j}$. Time separations: $\tau=-3.49 D_{j} / U_{j}$ (top row), $\tau=0$ (middle row), and $\tau=3.49 D_{j} / U_{j}$ (bottom row). Red line: SPS. White line: radiator surface. Black dashed vertical line: positions for a downstream convection at $0.6 U_{j}$. Color scale ranges from $\mathbf{- 0 . 7 5}$ (blue) to $+\mathbf{0 . 7 5}$ (red). 


\section{Concluding Remarks}

We used highly-resolved LES of a Mach 0.9 isothermal turbulent jet to investigate the connection between the statistics of the vortical field and its pressure signature on the edge of the jet. The representative locations for those regions are the surface of peak Reynolds stress (SPS) and the radiator surface, respectively. The definition of the radiator surface introduced in previous works [11] as the location where the convective velocity equals that on the SPS is used here. The axial and azimuthal scales of the flow field are calculated in the entire domain of relevance using two-point space-time correlations. The correlations are based on the axial velocity fluctuation $u^{\prime}$ and pressure fluctuation $p^{\prime}$. For the axial scales two definitions are considered, the fixed-frame scale $L$ and the moving-frame scale $\widehat{L}$.

The nature of the space-time correlations differs significantly for axial velocity fluctuations and pressure fluctuations. On the radial-axial plane, pressure-based correlations exhibit a wavepacket-like pattern that is coherent radially and travels fairly uniformly with the convective velocity $U_{c}$ on the SPS. On the other hand, the velocity-based correlation lacks strong radial coherence and its convection is non-uniform and biased by the mean-flow velocity. These results suggest that velocity-based correlations capture localized turbulent events, while the pressure-based correlations are dominated by the interaction of large eddies with the surrounding potential flow. Consequently, the axial and radial distributions of the corresponding length scales exhibit different trends. Pressure-based correlation scales on the radiator surface are larger than velocity- or pressure-based scales on the SPS, indicating that fine-scale vortical motions do not make a significant pressure imprint on the radiator surface.

Regarding axial trends, velocity-based length and azimuthal scales increase largely linearly with downstream distance, thus following the growth of the vortical region. The same holds for moving-frame length scales based on velocity. However, the moving-frame length scale based on pressure grows rapidly in the first few jet diameters, then saturates or grows slowly. The effect is most notable on the radiator surface. The azimuthal scale based on pressure also rises quickly and then saturates.

In the radial direction, starting from the centerline, axial scales based on velocity show a local maximum inside the vortical region and a local minimum near the edge of the jet. On the other hand, fixed-frame length scales based on pressure exhibit a monotonic increase up to a local maximum past the edge of the jet. Moving-frame scales based on pressure show a local maximum followed by a local minimum within the vortical region, and then rise to a higher maximum past the edge of the jet. The radial trends of fixed-frame length scales collapse well on a single curve when the radial coordinate is offset by the radial location of the inner edge of the shear layer and normalized by the momentum thickness. This works particularly well for axial locations of $x \geq 3 D_{j}$. The same scaling is carried out with moving-frame length scales and azimuthal scales, with equally good results with the exception of moving-frame length scales based on pressure whose growth does not follow that of the momentum thickness. A particularly interesting finding of the study is that the radiator surface passes through a "valley" (local minimum) of velocity-based length scale (fixed or moving-frame), indicating that the velocity fluctuations there are mostly decorrelated from events in the core vortical region.

Efforts are placed in connecting the LES-derived velocity scales and RANS-based scales on the SPS. The RANS values are calculated by "emulating" time-averaged turbulence kinetic energy and dissipation from the LES flow field. The RANS-based scales show good qualitative agreement with the velocity-based scales on the SPS, and thus give very similar distributions when fitted through a simple proportionality factor. The same attempt between pressure-based scales on the radiator and velocity-based scales on the SPS gives good results for fixed-frame length scales, but the trends suggest the need for non-linear relations when using moving-frame length scales and azimuthal length scales.

\section{Acknowledgment}

We gratefully acknowledge a Balsells Fellowship that partially supported A. Adam. 


\section{References}

[1] Lighthill, M., "On Sound Generated Aerodynamically: I. General Theory," Proceedings of the Royal Society of London, Vol. 211, 1952, pp. 564-587. https://doi.org/10.1098/rspa.1952.0060.

[2] Ffowcs Williams, J., and Hawkings, D., "Sound Generation by Turbulence and Surfaces in Arbitrary Motion," Philosophical Transactions of the Royal Society A, Vol. 264, No. 1151, 1969, pp. 321-342. https://doi.org/10.1098/ rsta.1969.0031.

[3] Pilon, A., and Lyrintzis, A., "Development of an Improved Kirchhoff Method for Jet Aeroacoustics," AIAA Journal, Vol. 36, No. 5, 1998, pp. 783-790. https://doi.org/https://doi.org/10.2514/2.437.

[4] Papamoschou, D., "Prediction of Jet Noise Shielding," AIAA Paper 2010-0653, 2010. https://doi.org/10.2514/6.2010653.

[5] Papamoschou, D., and Mayoral, S., "Modeling of Jet Noise Sources and their Diffraction with Uniform Flow," AIAA Paper 2013-0326, 2013. https://doi.org/https://doi.org/10.2514/6.2013-326.

[6] Papamoschou, D., "On the Connection Between Near and Far Pressure Fields of a Turbulent Jet," AIAA Paper 2018-1251, 2018. https://doi.org/10.2514/6.2018-1251

[7] Henderson, B., Leib, S., and Wernet, M., "Measurements and Predictions of the Noise from Three-Stream Jets," AIAA Paper 2015-3120, 2015. https://doi.org/10.2514/6.2015-3120.

[8] Phong, V., and Papamoschou, D., "Investigation of Isolated and Installed Three-Stream Jets from Offset Nozzles," AIAA Paper 2017-0005, 2017. https://doi.org/https://doi.org/10.2514/6.2017-0005.

[9] Papamoschou, D., "Wavepacket Modeling of the Jet Noise Source," International Journal of Aeroacoustics, Vol. 17, No. 1-2, 2018, pp. 52-69. https://doi.org/10.1177/1475472X17743653

[10] Adam, A., Papamoschou, D., Xiong, J., and Liu, F., "The Very Near Pressure Field of Three-Stream Jets," AIAA Paper 2018-1739, 2018. https://doi.org/10.2514/6.2018-1739.

[11] Adam, A., Papamoschou, D., Xiong, J., and Liu, F., "Vorticity Dynamics and Flow Statistics Near the Edge of HIgh Speed Multi-Stream Jets," AIAA Paper 2019-1303, 2019. https://doi.org/10.2514/6.2019-1303

[12] Harper-Bourne, M., "Jet Noise Turbulence Measurements," AIAA Paper 2003-3214, 2003. https://doi.org/10.2514/ 6.2003-3214.

[13] Morris, P., and Zaman, K., "Velocity Measurements in Jets with Application to Jet Noise," Journal of Sound and Vibration, Vol. 329, 2010, pp. 394-414. https://doi.org/10.1016/j.jsv.2009.09.024

[14] Proença, A., Lawrence, J., and Self, R., "Measurements of the single-point and joint turbulence statistics of high subsonic jets using hot-wire anemometry," Experiments in Fluids, Vol. 60, No. 4, 2019, p. 63. https://doi.org/10.1007/s00348-019-2716-3

[15] Bogey, C., Marsden, O., and Bailly, C., "Large-Eddy Simulation of the flow and acoustic fields of a Reynolds number $10^{5}$ subsonic jet with tripped exit boundary layers," Phys. Fluids, Vol. 23, No. 3, 2011, p. 035104. https://doi.org/10.1063/1.3555634

[16] Bogey, C., Marsden, O., and Bailly, C., "Influence of initial turbulence level on the flow and sound fields of a subsonic jet at a diameter-based Reynolds number of $10^{5}$," J. Fluid Mech., Vol. 701, 2012, pp. 352-385. https://doi.org/10.1017/jfm.2012.162.

[17] Bogey, C., "Grid sensitivity of flow field and noise of high-Reynolds-number jets computed by large-eddy simulation," Int. J. Aeroacoust., Vol. 17, No. 4-5, 2018, pp. 399-424. https://doi.org/10.1177/1475472X18778287

[18] Bogey, C., "Two-dimensional features of correlations in the flow and near pressure fields of Mach number 0.9 jets," AIAA Paper 2019-0806, 2019. https://doi.org/10.2514/6.2019-0806 
[19] Zaman, K. B. M. Q., "Effect of initial condition on subsonic jet noise," AIAA Journal, Vol. 23, No. 9, 1985, pp. 1370-1373. https://doi.org/10.2514/3.9094.

[20] Bogey, C., and Bailly, C., "Influence of nozzle-exit boundary-layer conditions on the flow and acoustic fields of initially laminar jets," Journal of Fluid Mechanics, Vol. 663, 2010, p. 507-538. https://doi.org/10.1017/ S0022112010003605

[21] Bogey, C., Marsden, O., and Bailly, C., "On the spectra of nozzle-exit velocity disturbances in initially nominally turbulent, transitional jets," Physics of Fluids, Vol. 23, No. 9, 2011, p. 091702. https://doi.org/10.1063/1.3642642.

[22] Bogey, C., Marsden, O., and Bailly, C., "Effects of moderate Reynolds numbers on subsonic round jets with highly disturbed nozzle-exit boundary layers," Physics of Fluids, Vol. 24, No. 10, 2012, p. 105107. https://doi.org/10.1063/1.4757667.

[23] Bogey, C., and Marsden, O., "Identification of the effects of the nozzle-exit boundary-layer thickness and its corresponding Reynolds number in initially highly disturbed subsonic jets," Physics of Fluids, Vol. 25, No. 5, 2013, p. 055106. https://doi.org/10.1063/1.4807071.

[24] Mohseni, K., and Colonius, T., "Numerical Treatment of Polar Coordinate Singularities," Journal of Computational Physics, Vol. 157, No. 2, 2000, pp. 787 - 795. https://doi.org/10.1006/jcph.1999.6382.

[25] Bogey, C., de Cacqueray, N., and Bailly, C., "Finite differences for coarse azimuthal discretization and for reduction of effective resolution near origin of cylindrical flow equations," Journal of Computational Physics, Vol. 230, No. 4, 2011, pp. 1134 - 1146. https://doi.org/10.1016/j.jcp.2010.10.031

[26] Bogey, C., and Bailly, C., "A family of low dispersive and low dissipative explicit schemes for flow and noise computations," Journal of Computational Physics, Vol. 194, No. 1, 2004, pp. 194 - 214. https://doi.org/10.1016/j. jcp.2003.09.003.

[27] Bogey, C., de Cacqueray, N., and Bailly, C., "A shock-capturing methodology based on adaptative spatial filtering for high-order non-linear computations," Journal of Computational Physics, Vol. 228, No. 5, 2009, pp. 1447 1465. https://doi.org/10.1016/j.jcp.2008.10.042.

[28] Bogey, C., and Bailly, C., "Three-dimensional non-reflective boundary conditions for acoustic simulations: Far field formulation and validation test cases," Acta Acustica united with Acustica, Vol. 88, 2002, pp. 463-471.

[29] Tam, C. K., and Dong, Z., "Radiation and outflow boundary conditions for direct computation of acoustic and flow disturbances in a nonuniform mean flow," Journal of computational acoustics, Vol. 04, No. 02, 1996, pp. 175-201. https://doi.org/10.1142/S0218396X96000040.

[30] Bogey, C., and Bailly, C., "Large eddy simulations of transitional round jets: Influence of the Reynolds number on flow development and energy dissipation," Physics of Fluids, Vol. 18, No. 6, 2006, p. 065101. https://doi.org/10.1063/1.2204060.

[31] Bogey, C., and Bailly, C., "Turbulence and energy budget in a self-preserving round jet: direct evaluation using large eddy simulation," Journal of Fluid Mechanics, Vol. 627, 2009, p. 129-160. https://doi.org/10.1017/ S0022112009005801.

[32] Fauconnier, D., Bogey, C., and Dick, E., "On the performance of relaxation filtering for large-eddy simulation," Journal of Turbulence, Vol. 14, No. 1, 2013, pp. 22-49. https://doi.org/10.1080/14685248.2012.740567.

[33] Kremer, F., and Bogey, C., "Large-eddy simulation of turbulent channel flow using relaxation filtering: Resolution requirement and Reynolds number effects," Computers \& Fluids, Vol. 116, 2015, pp. 17 - 28. https://doi.org/10. 1016/j.compfluid.2015.03.026.

[34] Dahl, M., "Turbulent Statistics from Time-Resolved PIV Measurements of a Jet Using Empirical Mode Decomposition," AIAA Paper 2012-2297, 2012. https://doi.org/10.2514/6.2012-2297. 
[35] Kerhervé, F., Fitzpatrick, J., and Jordan, P., "The frequency dependence of jet turbulence for noise source modelling," Journal of Sound and Vibration, Vol. 296, No. 1, 2006, pp. 209 - 225. https://doi.org/https: //doi.org/10.1016/j.jsv.2006.02.012.

[36] Panchapakesan, N. R., and Lumley, J. L., "Turbulence measurements in axisymmetric jets of air and helium. Part 1. Air jet," Journal of Fluid Mechanics, Vol. 246, 1993, p. 197-223. https://doi.org/10.1017/S0022112093000096

[37] Papamoschou, D., "Modelling of Noise Reduction in Complex Multistream Jets," Journal of Fluid Mechanics, Vol. 834, 2018, pp. 555-599. https://doi.org/10.1017/jfm.2017.730.

[38] Papamoschou, D., and Phong, V., "The Very Near Pressure Field of Single- and Multi-Stream Jets," AIAA Paper 2017-0230, 2017. https://doi.org/10.2514/6.2017-0230

[39] Papamoschou, D., Xiong, J., and Liu, F., “Towards a Low-Cost Wavepacket Model of the Jet Noise Source," AIAA Paper 2015-1006, 2015. https://doi.org/10.2514/6.2015-1006

[40] Picard, C., and Delville, J., "Pressure velocity coupling in a subsonic round jet," International Journal of Heat and Fluid Flow, Vol. 21, No. 3, 2000, pp. 359 - 364. https://doi.org/10.1016/S0142-727X(00)00021-7

[41] Coiffet, F., Jordan, P., Delville, J., Gervais, Y., and Ricaud, F., "Coherent Structures in Subsonic Jets: A Quasi-Irrotational Source Mechanism?" International Journal of Aeroacoustics, Vol. 5, No. 1, 2006, pp. 67-89. https://doi.org/10.1260/147547206775220407

[42] Kolár, V., "Brief Notes on Vortex Identification," Proceedings of the 8th WSEAS International Conference on Fluid Mechanics, 8th WSEAS International Conference on Heat and Mass Transfer, World Scientific and Engineering Academy and Society (WSEAS), Stevens Point, Wisconsin, USA, 2011, p. 23-28. 